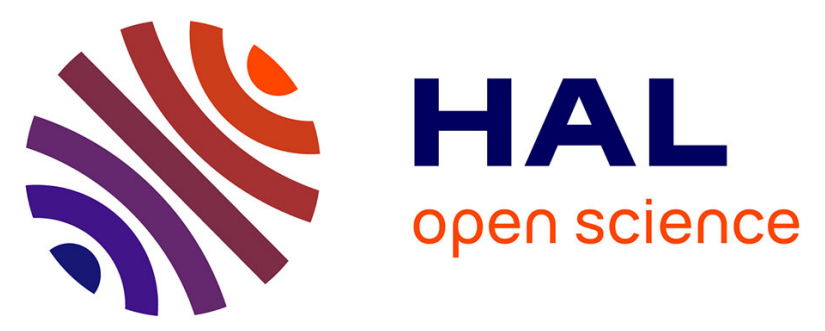

\title{
LYN-mediated mitochondrial tyrosine phosphorylation is required to preserve mitochondrial integrity in early liver regeneration
}

Enrico Gringeri, Amedeo Carraro, Elena Tibaldi, Francesco E d'Amico, Mario Mancon, Antonio Toninello, Mario Angelo Pagano, Claudia Vio, Umberto Cillo, Anna Maria Brunati

\section{To cite this version:}

Enrico Gringeri, Amedeo Carraro, Elena Tibaldi, Francesco E d'Amico, Mario Mancon, et al.. LYNmediated mitochondrial tyrosine phosphorylation is required to preserve mitochondrial integrity in early liver regeneration. Biochemical Journal, 2009, 425 (2), pp.401-412. 10.1042/BJ20090902 . hal00479212

\section{HAL Id: hal-00479212 https://hal.science/hal-00479212}

Submitted on 30 Apr 2010

HAL is a multi-disciplinary open access archive for the deposit and dissemination of scientific research documents, whether they are published or not. The documents may come from teaching and research institutions in France or abroad, or from public or private research centers.
L'archive ouverte pluridisciplinaire $\mathbf{H A L}$, est destinée au dépôt et à la diffusion de documents scientifiques de niveau recherche, publiés ou non, émanant des établissements d'enseignement et de recherche français ou étrangers, des laboratoires publics ou privés. 


\section{LYN-MEDIATED MITOCHONDRIAL TYROSINE PHOSPHORYLATION IS REQUIRED TO PRESERVE MITOCHONDRIAL INTEGRITY IN EARLY LIVER REGENERATION.}

Enrico Gringeri ${ }^{1,4}$, Amedeo Carraro ${ }^{2,4}$, Elena Tibaldi ${ }^{3}$, Francesco E D’Amico ${ }^{1}$, Mario Mancon ${ }^{3}$, Antonio Toninello ${ }^{3}$, Mario Angelo Pagano ${ }^{3, *}$, Claudia Vio $^{3}$, Umberto Cillo ${ }^{1,5}$ and Anna Maria Brunati ${ }^{3,5}$

${ }^{1}$ Department of General Surgery and Organ Transplantation, Hepatobiliary and Liver Transplant Unit, University of Padova, Via Giustiniani 2, 35128, Padova, Italy, ${ }^{2}$ Chirurgia Oncologica, IOV Istituto Oncologico Veneto, IRCCS, Via Gattamelata 64, 35128, Padova, Italy, ${ }^{3}$ Department of Biochemistry, University of Padova, Viale G. Colombo 3, 35131, Padova, Italy

Footnote : ${ }^{4}$ These authors contributed equally to this work, ${ }^{5}$ These authors are co-senior authors.

Keywords: apoptosis, mitochondrial membrane potential, oxidative stress, calcium overload.

Running Title: Lyn moves to mitochondria in early liver regeneration

*Corresponding author: M A Pagano,
Department of Biochemistry, University of Padova, Viale G. Colombo 3, 35131, Padova, Italy,
Tel: $+39-049-8276114$
Fax: $+39-049-8073310$
E-mail: mario.pagano@unipd.it

List of Abbreviations: liver regeneration, LR; partial hepatectomy, $\mathrm{PH}$; reactive oxygen species, ROS; oxidized/reduced glutathione, GSSG/GSH; src family kinases, SFKs; lamda phosphatase, $\lambda$ PPase; sodium dodecyl sulfate polyacrylamide gel electrophoresis, SDS-PAGE; mitochondrial membrane potential, $\Delta \Psi m$; Signal Transducer and Activator of Transcription-3, STAT3; Extracellular signal-Regulated Kinase-1/2, ERK-1/2; poly-ADP-ribose polymerase, PARP; phosphorylated Tyr396, P-Y A Apoptosis-Inducing Factor, AIF; cytochrome c oxidase, COX; adenine nucleotide translocase, ANT. 


\section{SYNOPSIS}

Functional alterations in mitochondria such as overproduction of reactive oxygen species and overloading of calcium, with subsequent change in the membrane potential, are traditionally regarded as pro-apoptotic conditions. Although such events occur in the early phases of liver regeneration after $2 / 3$ partial hepatectomy, hepatocytes do not undergo apoptosis but continue to proliferate until the mass of the liver is restored. The aim of this study was to establish whether tyrosine phosphorylation, an emerging mechanism of regulation of mitochondrial function, participates in the response to liver injury following $\mathrm{PH}$ and is involved in contrasting mitochondrial pro-apoptotic signalling. Mitochondrial tyrosine phosphorylation, negligible in the quiescent liver, was detected in the early phases of liver regeneration with a trend similar to the events heralding mitochondrial apoptosis and was attributed to the tyrosine kinase Lyn, a member of the Src family. Lyn was shown to accumulate in an active form in the mitochondrial intermembrane space, where it was found to be associated with a multiprotein complex. Our results highlight a role for tyrosine phosphorylation in accompanying, and ultimately counteracting, mitochondrial events otherwise leading to apoptosis, hence conveying information required to preserve the mitochondrial integrity during liver regeneration.

\section{INTRODUCTION}

Mitochondria are recognized to have a crucial role in several cellular functions. In addition to serving as the "powerhouse" of the cell to supply the majority of cellular ATP, mitochondria directly participate in cell metabolism, $\mathrm{Ca}^{2+}$ homeostasis, cell proliferation and death, the regulation of which requires integrated systems of signals that converge on these organelles. In this respect, signalling cascades generated at the cell membrane by hormones, growth factors, and cytokines target mitochondria and modulate their activity, although the molecular mechanisms are not fully understood [1-3]. Nevertheless, compelling evidence has been provided that reversible protein phosphorylation, the commonest post-translational modification, is one of the key regulatory mechanisms affecting most of, if not all, mitochondrial processes, including electron transport, Krebs cycle, fatty acids $\beta$-oxidation cycle, urea cycle, permeability transition and metabolite transport [4-9]. So far, a number of protein kinases, desides protein phosphatases, were found to be localized in all mitochondrial compartments, most of these also exerting a role outside mitochondria such as protein kinase $\mathrm{A}, \mathrm{B}$, and $\mathrm{C}$ (PKA, PKB/Akt and PKC), mitogen-activated

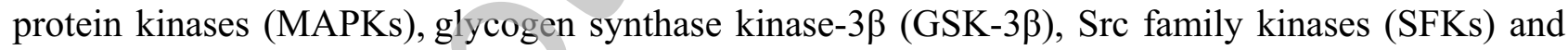
EGFR [10-14], suggesting a mechanism of translocation into mitochondria due to appropriate signals. Recently, we demonstrated that stimuli triggering cell proliferation brought about an elevation of tyrosine phosphorylation inside mitochondria. Such post-translational modification was shown to be bound to the presence of activated SFKs, a subgroup of non-receptor tyrosine kinases, traditionally considered as switch molecules beneath the plasma membrane capable of coupling receptor signals to downstream signaling pathways [15].

In order to further investigate the role of mitochondrial tyrosine phosphorylation during cell proliferation, we employed liver regeneration (LR) after 2/3 partial hepatectomy (PH) which is considered as an excellent in vivo model for studying cell cycle progression and cell proliferation [16]. In response to this surgical procedure, the remnant hepatocytes synchronously re-enter the cell cycle to undergo one to two rounds of replication before returning to the quiescent state, eventually restoring the mass and ensuring maintenance of the multiple functions of the liver [17-18]. The entry of quiescent hepatocytes into the cell cycle, corresponding to the G0/G1 transition, is largely regulated by cytokines, such as TNF- $\alpha$ and IL-6, leading to the activation of transcription factors like activator protein 1 (AP-1), Signal Transducer and Activator of Transcription-3 (STAT3) and Nuclear factor-kappaB $(\mathrm{NF}-\kappa \mathrm{B})$, followed by the transcription of genes encoding cell cycle 
regulators such as cyclin D [18]. This phase, which is reversible, primes hepatocytes to respond to growth factors, such as Hepatocyte Growth Factor (HGF) and Transforming Growth Factor Alpha (TGF- $\alpha$ ), in turn triggering signaling pathways that account for the up-regulation of a number of factors essential for the G1/S progression including cyclin E and cyclin $A$ and their respective cyclin-dependent kinases [18]. During this series of events, referred to as the pre-replicative phase, mitochondrial energy metabolism is impaired with an overproduction of reactive oxygen species (ROS) and subsequent increase in the mitochondrial oxidized/reduced glutathione (GSSG/GSH) as well as a decrease in the respiratory control index and in the rate of the oxidative phosphorylation [19-21]. Furthermore, accumulation of $\mathrm{Ca}^{2+}$ in mitochondria is concomitant with oxidative stress and both these phenomena are described to promote mitochondrial permeability transition in isolated mitochondria [21-22]. Despite these biochemical events, usually regarded as pro-apoptotic, neither release of cytochrome $\mathrm{c}$ nor appearance of apoptotic nuclei occur, confirming that hepatocytes do not undergo apoptosis during the prereplicative phase of LR and suggesting that mitochondria receive intracellular signals aimed at maintaining their structural and functional integrity and ultimately sustaining cell proliferation.

The aim of this work was to assess whether the signalling pathways activated by cytokines and growth factors during the early phases of LR also convey signals that modulate mitochondrial functions by involvement of tyrosine phosphorylation.

Here, we demonstrate that mitochondrial tyrosine phosphorylation, negliglible in the quiescente liver, increased and displayed a trend similar to that of oxidative stress, $\mathrm{Ca}^{2+}$ overloading and transmembrane hyperpolarization in the early phases of LR. Moreover, a key player in the elevation of mitochondrial tyrosine phosphorylation is shown to be the tyrosine kinase Lyn, a member of the SFKs, which accumulates in an active form in mitochondria and contributes to preserve mitochondrial integrity from the damage caused by liver injury, thus preventing hepatocytes from entering apoptosis.

\section{Materials}

\section{EXPERIMENTAL}

RNeasy Mini Kit was provided by Qiagen (Milan, Italy). Superscript II reverse transcriptase was from Invitrogen, (Carlsbad, CA, USA). Polymer polyGlu 4 Tyr, OptiPrep and phosphatase inhibitor cocktail 1 and 2 (PTP-I) were from Sigma-Aldrich (St. Louis, MO). [ $\gamma^{33}$ P]ATP was obtained from Perkin Elmer (NJ, USA). Protease inhibitor cocktail was from Roche.

Anti-phospho-SFK (Tyr-416) (pYA), anti-phospho-ERK, anti-ERK and anti-PARP polyclonal antibodies were from Cell Signaling Technology (Danvers, MA). Anti-Lyn, anti-cytochrome c, anti-cyclin D1, anti-cyclin E were from Santa Cruz Biotechnology (Santa Cruz, CA). The monoclonal anti-phosphotyrosine (clone PY-20) was from BioSource International (Camarillo, CA). Anti-phospho-STAT3, anti-STAT3 antibody were from Upstate Biotechnology (Lake Placid, NY). Anti- $\beta$-actin antibody (clone AC-15) was from Sigma-Aldrich. Anti-aconitase, anti-AIF and anti-Lactate Dehydrogenase (LDH) were from Abcam (Cambridge, MA)

The protein kinase inhibitors and analogs PP2, PP3, SU6656, piceatannol, AG490, AG1296, AG1478, genistein were purchased from Calbiochem (San Diego, CA). SU11274 was obtained from SUGEN, Inc. (South San Francisco, CA, USA). ECL detection system was from GE Healthcare (Little Chalfont, Buckinghamhsire, United Kingdom). Glutathione Assay Kit (GSH, GSSG and Total) was obtained from BioVision Inc. (Mountain View, CA).

\section{Partial hepatectomy}

Three-month old male Winstar rats $(250 \mathrm{~g}$ each) were purchased from Charles River Laboratories and were housed in the Animal Research Facility of Department of Biological-Chemistry. They 
were maintained under a 12-hour light-dark cycle and given rat chow and water ad libitum. Rats were subjected to $70 \% \mathrm{PH}$ [16] under isoflurane anesthesia and allowed to recover for various periods. Sham-operated rats, obtained by making small mid-line abdominal incision without excision of the liver, were used as a control and reported as time point $0 \mathrm{~h}$ in the experimental protocols. As to sham surgeries, livers were externalized and gently palpated to mimic the surgical stress of the $\mathrm{PH}$ procedure. One set of rats was alternatively treated with increasing concentrations of PP2, a potent and selective inhibitor for SFKs, and PP3, an inactive analog for PP2. Such molecules were dissolved in dimethylsulfoxide (DMSO) and further diluted in normal saline (final DMSO concentration was $0.5 \%$ ). PP2, PP3 or $0.5 \%$ DMSO in saline as vehicle control were injected intraperitoneally 4 hours before, immediately after and 12 hours following PH as described in [23]. Rats were sacrificed at $0,12,24,36$ and $48 \mathrm{~h}$ postoperatively; the livers were removed, weighed, and processed for distinct experimental purposes at the time-points indicated throughout the text. All operations were performed under sterile conditions. All animal studies were performed under animal care and use committee protocols approved by the State Commission on Animal Experimentation.

\section{Preparation of mitochondria}

Rat liver was homogenized in isolation medium (250 mM sucrose, $5 \mathrm{mM}$ Hepes, $0.5 \mathrm{mM}$ EGTA, $\mathrm{pH} 7.4)$ and subjected to centrifugation $(900 \mathrm{xg})$ for $5 \mathrm{~min}$. The supernatant was centrifuged at $12,000 \mathrm{x} g$ for $10 \mathrm{~min}$ to precipitate crude mitochondrial pellets. The pellets were resuspended in isolation medium plus $1 \mathrm{mM}$ ATP and layered on top of a discontinuous gradient of Ficoll diluted in isolation medium, composed of 2-ml layers of $16(\mathrm{w} / \mathrm{v}), 14$, and $12 \%$ Ficoll and a $3-\mathrm{ml}$ layer of $7 \%$ Ficoll. After centrifugation for $30 \mathrm{~min}$ at $75,000 \times \mathrm{g}$, mitochondrial pellets were suspended in isolation medium and centrifuged again for 10 min at $12,000 \times \mathrm{g}$. The resulting pellets were suspended in isolation medium without EGTA, and their protein content was measured by the biuret method, with bovine serum albumin as a standard. The absence of other contaminating subcellular compartments in our mitochondrial preparations has been demonstrated in previous studies [14].

\section{Subcellular fractionation}

Rat liver $(250 \mathrm{mg}$ ) was homogenized in $1 \mathrm{ml}$ of isolation medium (5 mM Hepes, pH 7.4, $250 \mathrm{mM}$ sucrose, protease inhibitor cocktail (Roche), phosphatase inhibitor cocktail I and II (Sigma) by 20 strokes with a Polytron tissue homogenizer and subjected to centrifugation for $10 \mathrm{~min}$ at $900 \mathrm{x} g$ (nuclei fraction, pellet I). The supernatant was then centrifuged for $1 \mathrm{~h}$ at $100,000 \times \mathrm{g}$ to separate cytosol from the post-nuclear particulate fraction (pellet II). The particulate fraction, resuspended in $200 \mu \mathrm{l}$ of the above described isotonic buffer, was overlaid onto a discontinuous gradient composed of 30, 25, 20, 15, and 10\% Optiprep ${ }^{\mathrm{TM}}$ (Accurate Chemical and Scientific Corp.).and dissolved in a

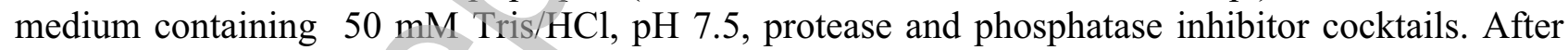
centrifugation at $100,000 \times \mathrm{g}$ for $3 \mathrm{~h}$ at $4{ }^{\circ} \mathrm{C}$, the gradient was divided into 15 equal aliquots collected from its top.

\section{Immunoprecipitation}

Liver tissue and and mitochondria were homogenized in a Dounce homogenizer (20 strokes) in buffer A (20 mM Tris-HCl, pH 7.4, 1mM EDTA, phosphatase inhibitor cocktail I and II, protease inhibitor cocktail) and suspended for 1 hour at $4{ }^{\circ} \mathrm{C}$ after supplementing the lysates with $1 \%$ Triton $\mathrm{X}-100)$. After centrifugation at $20000 \mathrm{x} \mathrm{g}$, supernatants were incubated overnight in the presence of anti-Lyn antibody and thereafter with protein A sepharose beads for $2 \mathrm{~h}$ at $4^{\circ} \mathrm{C}$. The protein-bead complex was centrifuged, washed and solubilized as detailed below.

\section{Proteinase K Treatment}

Purified mitochondria were treated with $50 \mathrm{ng} / \mathrm{ml}$ proteinase $\mathrm{K}$ in isolation medium without EGTA (see preparation of mitochondria) in the absence or presence of $0.5 \%$ Triton X-100 at room 
temperature for $30 \mathrm{~min}$. The reaction was stopped by the addition of protease inhibitor cocktail, and then analyzed by Western blotting with anti- Lyn, anti-aconitas and anti-AIF antibodies.

\section{Mitochondrial subfractionation}

To separate mitochondrial membranes from the soluble fractions, $5 \mathrm{mg}$ of mitochondria suspended in $250 \mathrm{mM}$ sucrose, $5 \mathrm{mM}$ Hepes, $0.5 \mathrm{mM}$ EGTA, pH 7.4 were sonicated in an MSE Sonicator and subjected to eight freeze/thaw cycles. Mitochondrial suspensions were then centrifuged at 100,000 $\mathrm{g}$ to obtain membrane pellets and supernatant fractions.

\section{Digitonin treatment}

Purified mitochondria $(1 \mathrm{mg} / \mathrm{ml})$ were incubated with increasing concentrations of digitonin (from 0.1 to $0.6 \mathrm{mg} / \mathrm{ml}$ ) for $30 \mathrm{~min}$. at $4{ }^{\circ} \mathrm{C}$, after which the samples were centrifuged at $22,800 \mathrm{x} \mathrm{g}$ for 20 min. Supernatant $(\mathrm{S})$ and pellet $(\mathrm{P})$ were subjected to $10 \%$ SDS-PAGE and Western blotting analysis with the appropriate antibody.

\section{Fractionation by centrifugation on glycerol gradient}

$150 \mu \mathrm{g}$ of soluble protein from rat liver at $24 \mathrm{~h}$ of regeneration were loaded on a 3.9-ml glycerol (10$40 \%$ ) linear gradient in $25 \mathrm{mM}$ Hepes, $\mathrm{pH} 7.4,1 \mathrm{mM}$ EDTA. The samples were centrifuged $18 \mathrm{~h}$ at $100,000 \mathrm{x} \mathrm{g}$ in a SW60Ti rotor (Beckman) at $4^{\circ} \mathrm{C}$, and divided into 18 equal aliquots collected from its top. Thyroglobulin $(669 \mathrm{kDa})$, apoferritin $(443 \mathrm{kDa})$, alcohol dehydrogenase $(150 \mathrm{kDa})$ and glutamate dehydrogenase $(62 \mathrm{kDa})$ were used as standards for estimating the MW of protein complexes.

\section{Western blot analysis}

Samples from total homogenates (rat liver, brain and spleen), different cell fractions (rat liver) and immunoprecipitates, were rapidly solubilized in $62 \mathrm{mM}$ Tris/HCL buffer, $\mathrm{pH} 6.8,5 \%$ glycerol and $0.5 \% \beta$-mercaptoethanol containing $0.5 \%$ SDS, run on $10 \%$ SDS-PAGE and transferred to nitrocellulose membranes by electroblotting. After treatment with $3 \%$ bovine serum albumin at $4^{\circ} \mathrm{C}$ overnight, membranes were incubated with the primary antibodies for $2 \mathrm{~h}$ and, after washing, with secondary horseradish peroxidase-conjugated polyclonal antibody for $1 \mathrm{~h}$. Immunoblots were developed by an enhanced chemiluminescence (ECL) detection system, captured using a Kodak Image Station 2000R and visualized by the Kodak 1D Image software (Rochester, NY). Membranes were reprobed with appropriate primary antibodies after stripping in $0.1 \mathrm{M}$ glycine ( $\mathrm{pH} 2.5), 0.5 \mathrm{M}$ $\mathrm{NaCl}, 0.1 \%$ Tween $20,1 \% \beta$-mercaptoethanol and $0.1 \% \mathrm{NaN}_{3}$ for $2 \times 10 \mathrm{~min}$ as loading control .

\section{Determination of mitochondrial calcium content.}

Purified mitochondria were solubilized to $1 \mathrm{mg} / \mathrm{ml}$ in $1 \mathrm{mM}$ EDTA, $0.1 \% \mathrm{NaCl}$ and $1 \%$ sodium deoxycholate. The calcium content in the resulting solution was estimated by atomic absorption using a Perkin Elmer AAnalyst 100 spectrophotometer.

\section{Measurements of GSH and GSSG}

GSH and GSSG were measured in isolated mitochondria by using Glutathione Assay Kit (GSH, GSSG and Total) (BioVision, Inc. Mountain View, CA).

\section{Determination of mitochondrial membrane potential $(\Delta \Psi \mathbf{m})$}

Mitochondrial membrane potential $(\Delta \Psi m)$ was estimated from the distribution of tetraphenylphosphonium $\left(\mathrm{TPP}^{+}\right)$measured across the mitochondrial membrane with a selective electrode prepared in our laboratory according to published procedures [14].

\section{Phosphorylation Assays}

Tyrosine phosphorylation was assayed on samples from total homogenate, mitochondrial lysates and Optiprep ${ }^{\mathrm{TM}}$ fractions by incubating $15 \mu \mathrm{g}$ of liver homogenate or liver mitochondria at $30^{\circ} \mathrm{C}$ for $10 \mathrm{~min}$ in $30 \mu \mathrm{l}$ of reaction medium containing $50 \mathrm{mM}$ Tris, $\mathrm{pH} 7.5,10 \mathrm{mM} \mathrm{MnCl}, 20 \mu \mathrm{M}[\mathrm{Y}$ - 
$\left.{ }^{33} \mathrm{P}\right]$ ATP $\left(3 \times 10^{6} \mathrm{cpm} / \mathrm{nmol}\right)$ and $1 \mathrm{mg} / \mathrm{ml}$ random polymer poly $(\mathrm{Glu}-\mathrm{Tyr})$ 4:1 (Sigma-Aldrich) or 200 $\mu \mathrm{M}$ cdc2 peptide, alternatively, which served as exogenous substrates in the absence and presence of specific tyrosine kinase inhibitors, i. e. SU11274 $(2 \mu \mathrm{M})$, AG1478 $(20 \mu \mathrm{M})$, AG1296 $(20 \mu \mathrm{M})$, AG490 $(20 \mu \mathrm{M})$, PP2 $(7.5 \mu \mathrm{M})$, SU6656 $(7.5 \mu \mathrm{M})$, piceatannol $(15 \mu \mathrm{M})$ or genistein $(20 \mu \mathrm{M})$. After incubation, samples were analyzed on $10 \%$ SDS-PAGE and revealed by a Cyclone Storage Phosphor Imager (Packard).

\section{RT-PCR analysis of Lyn, Src and $\beta$-actin transcripts}

Total RNA from rat regenerating livers at different time points after $\mathrm{PH}$ were isolated using Qiagen column kit (Qiagen). $2 \mu \mathrm{g}$ of RNA from each samples were reverse transcribed and the cDNA samples were then divided and amplified using specific primers $(20 \mathrm{pmoles} /$ tube). Primers were as follows: sense 5'- GGC TGA AGC CTC TGT CAT GAC GCA -3', antisense 5'- GCG TCT ACA CTA CAG GCG TGA CAG -3' for Lyn; sense 5'- GGC TGA AGC CTC TGT CAT GAC GCA -3', antisense 5'- GTC GAG GAC TTC GGA ACT CTC TCT -3' for c-Src; sense 5'- GTG GGG CGC CCC AGG CAC CA-3', antisense 5'- GAA ATC GTG CGT GAC ATT AAG GAG -3' for $\beta$-actin. The reaction mixture consisted of $1.5 \mathrm{mM} \mathrm{MgCl}_{2}, 500 \mathrm{mM} \mathrm{KCl}, 200 \mu \mathrm{M}$ dNTPs, and $2.5 \mathrm{U}$ Taq polymerase. Reaction conditions were 30 seconds of denaturation at $94^{\circ} \mathrm{C}, 30$ seconds of annealing at $60^{\circ} \mathrm{C}$, and 40 seconds of extension of $72^{\circ} \mathrm{C} 30$ cycles for c-Src and Lyn, 25 cycles for $\beta$-actin followed by a final extension of 7 minutes at $72^{\circ} \mathrm{C}$. Aliquots of PCR products were separated by electrophoresis on $2 \%$ agarose gel and visualized by ethidium bromide staining.

\section{Statistical analysis}

The bands on Western blots were quantified by densitometric analysis. Data are presented as mean \pm SD and compared by one-way analysis of variance (ANOVA) followed by Bonferroni post hoc test. A p-value $<0.05$ was considered as statistically significant. All statistics were performed using GraphPad Prism (version 4) statistical software (GraphPad Software; San Diego, CA).

\section{RESULTS}

\section{Protein tyrosine phosphorylation increases in isolated mitochondria from rat regenerating liver after PH}

To establish whether mitochondrial tyrosine phosphorylation was implicated in the transient alterations of mitochondrial function usually observed during the early phase of LR after PH [2023], we purified mitochondria from liver of sham-operated and partially hepatectomized rats according to protocols elsewhere reported [14]. As shown in Figure 1A, mitochondrial tyrosine phosphorylation increased over a wide range of molecular weights in a time-dependent manner with a maximal peak between 24 and $36 \mathrm{~h}$ after $\mathrm{PH}$, subsequently declining to a basal value by $48 \mathrm{~h}$. The time interval at which mitochondrial tyrosine phosphorylation was maximal reflected progression from $\mathrm{G}_{1}$ - into $\mathrm{S}$-phase of the cell cycle, as assessed by monitoring the expression of both cyclin D1, whose up-regulation indicates the exit from $G_{0}$ into the $G_{1}$ restriction point, and of cyclin $E$, which is involved in the $\mathrm{G}_{1}-\mathrm{S}$ phase transition, by Western blot analysis on the total cell lysate (Figure 1B) [24,25]. We similarly detected phosphorylated STAT3 (P-STAT3) and phosphorylated Extracellular signal-Regulated Kinase-1/2 (P-ERK-1/2) (Figure 1B), which represent markers characterizing the cell cycle re-entry resulting from the cytokine- and growth factor-mediated response to liver injury [26-28]. Within the same time frame, we measured a few parameters of mitochondrial function, which are affected during LR, namely glutathione redox status (GSSG/GSH), concentration of $\mathrm{Ca}^{2+}$ and mitochondrial transmembrane potential $(\Delta \Psi \mathrm{m})[20-23]$. In accordance with previous reports, GSSG/GSH, an index of mitochondrial oxidative stress [29] and concentration of $\mathrm{Ca}^{2+}$, a key regulator of multiple mitochondrial functions acting at several levels [30], were increased about 5-fold and 2-fold, respectively, 24h after $\mathrm{PH}$, thereafter gradually 
decreasing, though not totally returning to the starting level, by $48 \mathrm{~h}$ (Figure $1 \mathrm{C}$, upper and middle panels). $\Delta \Psi \mathrm{m}$ rose to the maximal hyperpolarization after $24 \mathrm{~h}$ and subsequently declining to the basal value (Figure 1C, bottom panel). Besides, we also observed that cytochrome c was not released into the cytosol (Figure 1D) and that poly-ADP-ribose polymearse (PARP) cleavage did not occur (Figure 1E), thus underscoring the absence of any apoptotic event $[21,22]$.

\section{Src family kinases as major agents in mitochondrial tyrosine phosphorylation}

In an effort to identify the tyrosine protein kinase(s) responsible for the tyrosine phosphorylation detected in mitochondria during LR at $24 \mathrm{~h}$ after $\mathrm{PH}$, liver homogenates and lysates from highly purified mitochondria obtained from both liver of sham-operated and partially hepatectomized rats were tested for tyrosine kinase activity on a generic poly(Glu-Tyr) substrate in the absence and presence of various specific tyrosine kinase inhibitors. In particular, we used selective inhibitors of both receptor tyrosine kinases (SU11274, AG1478 and AG1296 against MET, EGFR and PDGFR respectively) [31-33] and of non-receptor tyrosine kinases (AG490, PP2 or, alternatively, SU6656 and piceatannol against JAK, SFKs and Syk respectively) [34-36] and genistein as a more generic compound active against both groups [37]. All the inhibitors assayed but AG1296 and Syk inhibitor significantly affected the tyrosine kinase activity in total cell lysates (Figure 2A), whereas only PP2 and SU6656, specific inhibitors of Src Family Kinases (SFKs), proved to be remarkably effective in inhibiting the tyrosine kinase activity in the mitochondrial lysate of regenerating livers (Figure 2B). These data corroborate the notion that SFKs are the major agents in mitochondrial tyrosine phosphorylation.

\section{Lyn is the predominant SFK expressed in the liver}

SFKs comprise eight non-receptor protein tyrosine kinases characterised by a common domain structure and grouped into two subfamilies based on their amino acid sequence, namely Src related (Src, Yes, Fyn and Fgr) and Lyn related SFKs (Lyn, Hck, Lck and Blk), respectively [38,39]. To date, few of them have been extensively studied as to their role in the liver, and mostly when implicated in carcinogenesis and, albeit only recently, in LR [40-42]. To assess which member of SFKs was responsible of the tyrosine kinase activity in mitochondria after $\mathrm{PH}$, we compared the protein level of SFKs in liver, spleen and brain, the two latter of which are organs abundantly expressing SFKs [40]. Equal amounts of protein lysate $(50 \mu \mathrm{g})$ from rat liver, spleen and brain underwent Western blot analysis with specific antibodies against SFKs and the resulting bands were quantified by densitometric analysis. As shown in Figure 3A, while Src related SFKs displayed a lower protein level in the liver than in spleen and brain, Lyn related ones were undetectable with the exception of Lyn, which turned out to be the predominant SFK expressed in the liver in comparison with the other two organs. This result was confirmed by RT-PCR, which revealed that mRNA levels of Lyn was 5-6 fold higher than those of c-Src analyzed in the quiescent liver. Notably, Lyn expression remained unchanged over time even after $\mathrm{PH}$, whereas the levels of c-Src were significantly lower than that of Lyn itself, albeit increased when compared to the basal value (Figure 3B).

\section{Lyn accumulates in mitochondria in an active form in early $L R$.}

Since Lyn was the predominant SFK in the liver, we investigated whether activity and protein level of Lyn in mitochondria differed in regenerating compared to quiescent liver. Therefore, we monitored SFK activity of both mitocondrial and total cell lysates by using the SFK-specific cdc2(6-20) peptide as a substrate. SFK activity increased already $12 \mathrm{~h}$ after $\mathrm{PH}$ in total cell lysate, substantially remaining unchanged at the next time-points (Figure 4A); instead, mitochondrial SFK 
activity, still negligible $12 \mathrm{~h}$ after $\mathrm{PH}$, dramatically rose at $24 \mathrm{~h}$, subsequently declining to a basal level by $48 \mathrm{~h}$ (Figure 4B). Western blot analysis of total cell lysate with anti-Lyn antibody demonstrated that the protein level of Lyn was not significantly altered during the regenerating process, confirming the results obtained by RT-PCR (Figure 4A); conversely, it was undetectable in mitochondria until $12 \mathrm{~h}$ after $\mathrm{PH}$, subsequently increasing to a peak between 24 and $36 \mathrm{~h}$, thereafter declining to the level reached at $12 \mathrm{~h}$ (Figure $4 \mathrm{~B}$ ). Since activity and protein level of Lyn were proportionally related to each other in the mitochondrial but not in the total cell lysate (Figure 4A vs 4B), we evaluated the activation state of Lyn by Western blot analysis against phosphorylated Tyr396 $\left(\mathrm{P}-\mathrm{Y}_{\mathrm{A}}\right)$, which is suggestive of the presence of an active form of Lyn [39]. Lyn immunoprecipitated from either total cell lysate or mitochondria of regenerating liver at different time-points was subjected to Western blot analysis against both $\mathrm{P}-\mathrm{Y}_{\mathrm{A}}$ and Lyn, revealing that the activation state followed a trend similar to that of the kinase activity (Figure 4A and 4B compared with 4C and 4D, respectively). Lyn appeared to be transiently localized to mitochondria in an active form during the early phases of LR after PH (Figure 4D). Notably, c-Src, Yes, Fyn and Fgr were not detected when tested for by Western blot analysis (data not shown). To verify how Lyn distribution changed during LR, the post-nuclear particulate obtained from either quiescent or regenerating liver 12 and $24 \mathrm{~h}$ after $\mathrm{PH}$ was centrifuged on a discontinuous OptiPrep ${ }^{\mathrm{TM}}$ gradient to separate the subcellular fractions. Lyn, abundantly represented on the plasma membrane in an inactive form in the quiescent liver (Figure 5A), appeared to be activated in this cellular compartment in the regenerating liver $12 \mathrm{~h}$ after $\mathrm{PH}$ (Figure 5B), with no change in the protein distribution. $24 \mathrm{~h}$ after $\mathrm{PH}$, we observed an increase in the SFK activity in the mitochondrial fractions concomitantly with the appearance of Lyn in the samel fractions and a decrease in the protein level of Lyn itself in the plasma membrane fractions (Figure 5C). These data suggest that Lyn, in response to liver injury, translocates and conveys information from the plasma membranes to, thus directly involving, mitochondria in LR.

\section{Lyn is detected in a complex in the intermembrane space of mitochondria.}

To establish in which mitochondrial subcompartment Lyn was localized, mitochondria isolated from regenerating liver $24 \mathrm{~h}$ after $\mathrm{PH}$ first underwent proteinase $\mathrm{K}$ treatment. As shown in Figure 6A, neither Lyn nor Apoptosis-Inducing Factor (AIF), a structural component of the inner mitochondrial membrane nor aconitase, a mitochondrial matrix protein, were processed by the protease treatment, which was instead effective on the three proteins only after total solubilisation with Triton X-100. Following separation of mitochondrial membranes and soluble fraction, Lyn as well as aconitase turned out to be predominantly localized in the soluble fraction, whereas AIF was in the membrane fraction (Figure 6B). Permeabilization with digitonin was also used to selectively permeabilize the outer mitochondrial membrane [43], causing a partial release of Lyn at a concentration of the detergent as low as $0.10 \mathrm{mg} / \mathrm{ml}$, which was fully achieved already at $0.20 \mathrm{mg} / \mathrm{ml}$ (Figure 6C). On the other hand, AIF and aconitase were nearly totally released into the soluble fraction only at concentrations of digitonin as high as $0.60 \mathrm{mg} / \mathrm{ml}$. Hence, Lyn was detected in the intermembrane space of mitochondria as a soluble protein as previously described in mitochondria of rat brain [14]. In an attempt to explain how Lyn, which is known to be bound to the plasma membrane by myristoylation of its $\mathrm{N}$-terminal, was targeted to mitochondria, the soluble submitocondrial fraction was run on a glycerol gradient. Lyn was detected by Western blot analysis in the correspondence of a molecular weight of $230 \mathrm{kDa}$, suggesting its binding to a multiprotein complex (Figure 6D).

\section{Lyn-mediated tyrosine phosphorylation preserves mitochondrial integrity.}

It is widely known that disruption of the mitochondrial membrane potential $(\Delta \Psi \mathrm{m})$ is often a consequence of oxidative stress and elevation of $\left[\mathrm{Ca}^{2+}\right]$ in mitochondria, with subsequent functional impairment and the activation of the apoptosis pathways. In Figure 1C, actually, we showed that the 
increased GSSG/GSH ratio and $\mathrm{Ca}^{2+}$ overload (upper and middle panel) were associated with a hyperpolarization, rather than a drop, of the inner membrane potential (bottom panel). Since the trend of all such functional parameters measured over time displayed a pattern analogous to that of tyrosine phosphorylation (Figure 1A), we examined whether this post-translational modification had a role in the behaviour of the mitochondrial membrane potential during LR. For this purpose, mitochondria purified from regenerating compared with quiescent liver were tested for $\Delta \Psi \mathrm{m} 24 \mathrm{~h}$ after $\mathrm{PH}$, which time-point corresponded to a peak of both tyrosine phosphorylation and the other parameters (Figure 1A and 1C). Notably, mitochondria were isolated and maintained in a medium containing protein tyrosine phosphatase inhibitors (PTP-I), consistently with the experimental protocol adopted throughout this work to emphasize phosphotyrosine signal, per se necessarily transient, or devoid of PTP-I themselves, so as not to rule out a possible role of phosphatases to counteract tyrosine kinase activity. Interestingly, mitochondria from quiescent liver did not differ in yield (data not shown), tyrosine phosphorylation pattern, the latter turning out to be virtually absent (Figure 7A lane 1 vs lane 2), and $\Delta \Psi \mathrm{m}$ pattern (Figure 7B, left panel), whether in the presence or absence of PTP-I. Mitochondria from regenerating liver in the absence of PTP-I, instead, displayed a significantly lower value of $\Delta \Psi \mathrm{m}$ than that of quiescent liver, finally collapsing after the addition of ADP, when the mitochondrial capability to perform oxidative phosphorylation was tested. Conversely, mitochondria from regenerating liver isolated in the presence of PTP-I showed a remarkable elevation of both tyrosine phosphorylation (Figure 7A, lane 3) and membrane polarization (Figure 7B, right panel) not only when compared with mitochondria without PTP-I from regenerating liver but also with those from quiescent liver. Moreover, the experimental conditions determined by the presence of PTP-I highlighted a conserved efficiency in oxidative phosphorylation, as confirmed by the transient effect on $\Delta \Psi \mathrm{m}$ upon addition of ADP, although the recovery of $\Delta \Psi \mathrm{m}$ was slower in mitochondria from regenerating than from quiescent liver (Figure $7 \mathrm{~B}$, right vs left panel), suggesting some residual activity that disappears within few minutes (data not shown).

An approach to establish how tyrosine phosphorylation, and namely that dependent on SFKs, impacted on the mitochondrial role in the early phases of LR consisted in the use of a specific SFK inhibitor, PP2, administered to rats undergoing PH. The effect of PP2 was assessed by investigating a few extra- and intramitochondrial events $24 \mathrm{~h}$ after $\mathrm{PH}$, that is, when the phosphorylation level was highest. Mitochondrial tyrosine phosphorylation, activation of Lyn and $\Delta \Psi \mathrm{m}$ (Figure $8 \mathrm{~A}, \mathrm{~B}$ and C respectively) all proved to be dose-dependently decreased in PP2-treated compared with rats treated with vehicle $(0.5 \%$ DMSO in saline). Interestingly, inhibition of Lyn (Figure 8B, upper panel) appeared to directly prevent the accumulation of Lyn itself in mitochondria (Figure 8B, lower) and consequently to account for the drop in mitochondrial tyrosine phosphorylation illustrated in Figure 8A. The collapse of $\Delta \Psi \mathrm{m}$ caused by increasing doses of PP2 (Figure $8 \mathrm{C}$ ) was a further finding indicating mitochondrial tyrosine phosphorylation as a determining factor for preserving the functional integrity of mitochondria in the early phases of LR. Notably, inhibition of SFK activity by PP2 resulted in a lower yield in intact mitochondria purified from rat liver (data not shown), as a result of disruption of mitochondrial membranes documented by the presence of cytochrome $\mathrm{c}$ and aconitase in the cytosolic fraction (Figure 8D). PP3, an inactive analog of PP2, at concentrations identical to those to PP2 or saline turned out to be incapable of affecting the mitochondrial events under investigation.

\section{DISCUSSION}

During the prereplicative phase of LR, mitochondria undergo functional changes that are traditionally considered as pro-apoptotic, such as $\mathrm{Ca}^{2+}$ overload and excessive production of ROS, though not leading to the activation of the death cascade. In this work, we provide evidence that the tyrosine kinase Lyn, a member of SFKs, accumulates in an active form in the intermembrane space 
of mitochondria and contributes to preserve the overall integrity of these organelles in the early phases of LR by phosphorylating a wide range of substrates.

SFKs are non-receptor tyrosine kinases that are co-expressed with multiple combinations of each member in various cell types. They serve as molecular switches involved in the integration and transmission of diverse signals generated by the triggering of cell surface receptors in response to a large number of extracellular stimuli, such as growth factors and cytokines, and resulting in a variety of cellular events, including cell growth and proliferation, cell adhesion and migration, differentiation, survival, and death [38-39]. SFKs are ordinarily maintained in a closed inactive conformation through two major intramolecular inhibitory interactions, and namely binding of the phosphorylated C-terminal tyrosine ( $\mathrm{Y}_{\mathrm{T}}, \mathrm{Tyr} 508$ of Lyn) to the $\mathrm{SH} 2$ domain, and interaction of a polyproline type II helical motif (PPII) in the SH2-kinase linker with the SH3 domain [44-45]. The activation of SFKs involves disruption of these inhibitory interactions through multiple mechanisms, such as dephosphorylation of $\mathrm{Y}_{\mathrm{T}}$, displacement of the tail from the $\mathrm{SH} 2$ domain, displacement of the PPII motif from the $\mathrm{SH} 3$ domain and is characterized by autophosphorylation of a specific tyrosine in the activation loop ( $\mathrm{Y}_{\mathrm{A}}, \mathrm{Tyr} 508$ of Lyn).

Although SFKs are generally believed to have redundant functions, emerging evidence indicates that each SFK member may possess unique roles in conferring signaling specificity owing to their ability to phosphorylate peculiar substrates, spatial compartmentalization in membrane microdomains and subcellular distribution $[39,46]$. Although most of the knowledge on SFKs have been gained by studies on the hematopoietic tissue, the presence and the role of SFKs and their substrates have recently been highlighted in other tissues and organs, among which the liver under normal and pathological conditions [40-42].

Our data show that, among the SFKs that are co-expressed with different levels of expression in the quiescent liver, Lyn proves to be quantitatively predominant. Moreover, although its expression remains unchanged during LR, Lyn undergoes spatio-temporal changes in the distribution and activity profile, as observed in the subcellular membrane fractions from liver homogenate. In particular, we observe an increase in the protein level of Lyn in an active form in mitochondria with a concurrent decrease at the plasma membrane, where the activation of the tyrosine kinase occurs in response to stimuli initiating LR following PH. Concurrently with a rise in SFK activity in the total liver lysate, no SFK member other than Lyn was detected in mitochondria either in the quiescent or in the regenerating liver (data not shown), indicating that Lyn is the only SFK member to be imported into mitochondria during LR. The data emerged in this work are in agreement with the role that Lyn possesses in the immune system, so far studied more deeply than the liver, where, in addition to exert functions redundant with other SFKs, it proves irreplaceable in the regulation of specific signalling pathways. For instance, Lyn not only contributes to initiate the signaling pathways upon ligation of the B cell receptor (BCR) redundantly with Blk and Fyn [47], but also negatively regulates the $\mathrm{BCR}$ signaling by phosphorylating inhibitory receptors, displaying a unique ability to modulate the a negative feedback control mechanism of signaling.

Activation of Lyn at the plasma membrane is shown to be conditio sine qua non for its accumulation in mitochondria, as demonstrated by the administration of PP2 (Figure 8B), inhibitor specific for SFKs in general but not for the single members of this kinase family, to rats in the early phases of LR. This finding allows to classify Lyn into a group of kinases that, upon external stimuli, are activated at the plasma membrane, such as EGFR, PKC and Akt, and are imported into mitochondria, where they impinge on mitochondrial function, thereby influencing cell survival [4850]. Recently, it has been demonstrated that the level of activated Akt in mitochondria is dependent on HSP90 chaperoning activity and that Akt import is involved in preserving mitochondrial integrity. [51]. Since we have recently demonstrated that, albeit under pathological conditions [52], Lyn is stabilized in an active form taking part in a multi-protein complex along with HSP90, we 
infer that the mechanism of import of Lyn may, similarly to Akt, rely upon the interaction with this chaperone, although further investigation is needed to verify this hypothesis.

Irrespective of how Lyn translocates into mitochondria, Lyn turns out to be responsible for the phosphorylation of numerous proteins inside these organelles during the pre-replicative phase of LR, since i) the temporal pattern of the level of activated Lyn in mitochondria (Figure 4B and 4D) is analogous to that of mitochondrial tyrosine phosphorylation (Figure 1A) and ii) administration of PP2 to rats undergoing PH results in a decrease in the protein level of Lyn in mitochondria that parallels the drop in mitochondrial tyrosine phosphorylation.

In support of the role of tyrosine phosphorylation in mitochondria, recent studies have revealed that several tyrosine phosphorylated proteins are found to be strategically localized in the various mitochondrial compartments and functionally affected by this post-translational modification [6]. For instance, the efficiency of the electronic-transport chain is enhanced when the subunit II of cytochrome c oxidase (COX) is phosphorylated by c-Src, whereas it is reduced when Tyr304 of subunit $\mathrm{I}$ is phosphorylated by a still unknown tyrosine kinase dependent on cAMP-mediated signaling $[4,6]$. Cytochrome $\mathrm{c}$ has also been shown to be regulated by the phosphorylation of Tyr97, resulting in inhibition of respiration in the reaction with COX. In addition, tyrosine phosphorylation is proposed to prevent the interaction of cytochrome c with Apaf-1, which would otherwise lead to apoptosome formation, and to inhibit the peroxidase activity responsible for cardiolipin oxidation: the two latter events are required for the release of cytochrome $\mathrm{c}$ into the cytosol together with other pro-apoptotic factors [6]. Tyrosine phosphorylation has also been taken into account in the regulation of adenine nucleotide translocase (ANT), the most abundant mitochondrial carrier that catalyzes the ADP/ATP exchange across the inner mitochondrial membrane and implicated in the formation or regulation of the mitochondrial permeability transition pore. We have lately identified two tyrosines of ANT, Tyr190 and Tyr194, phosphorylated by SFKs in mitochondria from rat brain [9]. Since these tyrosines are localized in the cavity where nucleotides bind for translocation, phosphorylation might represent a further mode of regulation of ANT activity, in addition to the activating mechanism dependent on oxidation of thiol groups. A recent study showed that phosphorylation of these residues is critical for mitochondrial bioenergetics and also associated with cardioprotection in a model of ischaemiareperfusion injury [53].

The latter finding is in agreement with our data, highlighting a role for tyrosine phosphorylation in preserving mitochondrial function observed during the early stage of LR despite the occurrence of possible pro-apoptotic processes, such as mitochondrial $\mathrm{Ca}^{2+}$ accumulation and oxidative stress, as confirmed by the absence of effects related to apoptogenic events, in particular the release of cytochrome $\mathrm{c}$ and the cleavage of PARP.

Notably, tyrosine phosphorylation was emphasized only by adding PTP-I in the media used to isolate mitochondria (Figure 7A, lane 3), to prevent dephosphorylation of mitochondrial proteins that regularly occurs during such procedures. The role of tyrosine phosphorylation in liver mitochondria under regenerating conditions was also stressed by the evidence that the absence of PTP-I is accompanied by a very low level of $\Delta \Psi \mathrm{m}$, in sharp contrast with the remarkable rise in $\Delta \Psi \mathrm{m}$ observed when PTP-I were used (Figure 7 , right panel). It is noteworthy that the $\Delta \Psi \mathrm{m}$ profiles of mitochondria from quiescent liver were identical regardless of the presence of PTP-I (Figure 7B, left panel). Another striking feature that further spotlights the importance of tyrosine phosphorylation in the processes here described is how this post-translational modification, by sustaining and even increasing $\Delta \Psi \mathrm{m}$, efficiently preserves ATP synthesis, which experimentally follows the addition of ADP to mitochondria isolated in the presence of PTP-I, very similarly to the resting state (Figure 7B, right vs left panel); on the other hand, mitochondria from regenerating liver without precautional treatment with PTP-I displayed not only a much lower $\Delta \Psi \mathrm{m}$ but also a 
ultimate collapse after addition of ATP, suggestive of an irreversible damage of the inner mitochondrial membrane. Treatment with PP2 of rats undergoing PH and subsequent LR displayed effects similar to those due to the absence of PTP-I on isolated mitochondria, in addition to an increased fragility of mitochondrial membranes as evidenced by the release of cytochrome c and aconitase in the cytosol (Figure 8D).

In summary, these data are consistent with a role for tyrosine phosphorylation mediated by Lyn in protecting the structural and consequently functional integrity as well as the bioenergetic competence of mitochondria by buffering the potentially harmful effects due to ROS and $\mathrm{Ca}^{2+}$ overload, otherwise leading to the activation of the death signaling, during the early phases of LR. In this respect, these findings warrant further efforts to broaden our knowledge on the role of mitochondrial tyrosine phosphorylated proteins and how their function is affected by this posttranslational modification. 


\section{REFERENCES}

1. Goldenthal MJ, Marín-García J. (2004) Mitochondrial signaling pathways: receiver/integrator organelle.Mol Cell Biochem. 262, 1-16.

2. Lai HC, Liu TJ, Ting CT, Sharma PM, Wang PH. (2003) Insulin-like growth factor- prevents loss of electrochemical gradient in cardiac muscle mitochondria via activation of PI 3 kinase/Akt pathway. Mol Cell Endocrinol. 205, 99-106.

3. Psarra AM, Solakidi S, Sekeris CE. (2006) The mitochondrion as a primary site of action of regulatory agents involved in neuroimmunomodulation. Ann N Y Acad Sci. 1088, 12-22.

4. Pagliarini, D.J. and Dixon, J.E. (2006) Mitochondrial modulation: reversible phosphorylation takes center stage? Trends Biochem. Sci. 31, 26-34

5. Salvi, M., Brunati, A.M. and Toninello, A. (2005) Tyrosine phosphorylation in mitochondria: a new frontier in mitochondrial signalling. Free Radic. Biol. Med. 38, 1267-1277

6. Hüttemann, M., Lee, I., Samavati, L., Yu, H. and Doan, J.W. (2007) Regulation of mitochondrial oxidative phosphorylation through cell signaling. Biochim. Biophys. Acta 1773, 1701-1720

7. Villen, J., Beausoleil, S.A., Gerber, S.A. and Gygi, S.P. (2007) Large-scale phosphorylation analysis of mouse liver. Proc. Natl. Acad. Sci. USA 104, 1488-1493

8. Lee, J., Xu, Y., Chen, R., Sprung, Y., Kim, S.C., Xie, S. and Zhao, Y. (2007) Mitochondrial phosphoproteome revealed by an improved IMAC method and MS/MS/MS, Mol. Cell. Proteomics 6, 669-676

9. Lewandrowski, U., Sickmann, A., Cesaro, L., Brunati, A.M., Toninello, A. and Salvi, M. (2008) Identification of new tyrosine phosphorylated proteins in rat brain mitochondria. FEBS Lett. 582, 1104-1110

10. Horbinski C, Chu CT. (2005) Kinase signaling cascades in the mitochondrion: a matter of life or death. Free Radic Biol Med. 38, 2-11

11. Yonekawa H, Akita Y. (2008) Protein kinase Cepsilon: the mitochondria-mediated signaling pathway. FEBS J. 275, 4005-13.

12. Parcellier A, Tintignac LA, Zhuravleva E, Hemmings BA. (2008) PKB and the mitochondria: AKTing on apoptosis. Cell Signal. 20, 21-30.

13. Salvi, M., Brunati, A.M., Bordin, L., La Rocca, N., Clari, G. and Toninello, A. (2002) Characterization and location of Src-dependent tyrosine phosphorylation in rat brain mitochondria. Biochim. Biophys. Acta. 1589, 181-195

14. Salvi, M., Stringaro, A., Brunati, A.M., Agostinelli, E., Arancia, G., Clari, G. and Toninello, A. (2004) Tyrosine phosphatase activity in mitochondria: presence of Shp-2 phosphatase in mitochondria. Cell. Mol. Life Sci. 61, 2393-2404

15. Tibaldi E, Brunati AM, Massimino ML, Stringaro A, Colone M, Agostinelli E, Arancia G, Toninello A. (2008) Src-Tyrosine kinases are major agents in mitochondrial tyrosine phosphorylation. J Cell Biochem. 104,840-849.

16. Higgins, G.M. and Anderson, R.M. (1931) Experimental pathology of the liver. Arch. Pathol. 12, 186-202

17. Taub, R. (2004) Liver regeneration: From myth to mechanism. Nat. Rev. Mol. Cell. Biol. 5, 836- 847

18. Fausto, N., Campbell, J.S. and Riehle, K.J. (2006) Liver regeneration. Hepatology 43, 45-53

19. Lee, F.Y., Li, Y., Zhu, H., Yang, S., Lin, H.Z., Trush, M. and Diehl, A.M. (1999) Tumor necrosis factor increases mitochondrial oxidant production and induces expression of uncoupling protein-2 in the regenerating mice [correction of rat] liver. Hepatology 29, 677687 
20. Guerrieri, F., Pellecchia, G., Lopriore, B., Papa, S., Esterina Liquori, G., Ferri, D., Moro, L., Marra, E. and Greco, M. (2002). Changes in ultrastructure and the occurrence of permeability transition in mitochondria during rat liver regeneration. Eur. J. Biochem. 269, 3304-3312

21. Ferri, D., Moro, L., Mastrodonato, M., Capuano, F., Marra, E., Liquori, G.E. and Greco, M. (2005) Ultrastructural zonal heterogeneity of hepatocytes and mitochondria within the hepatic acinus during liver regeneration after partial hepatectomy. Biol. Cell. 97, 277-288

22. Moro, L., Marra, E., Capuano, F. and Greco, M. (2004) Thyroid hormone treatment of hypothyroid rats restores the regenerative capacity and the mitochondrial membrane permeability properties of the liver after partial hepatectomy. Endocrinology 145, 5121-5128

23. Jiang X, Mu D, Biran V, Faustino J, Chang S, Rincón CM, Sheldon RA, Ferriero DM. (2008) Activated Src kinases interact with the N-methyl-D-aspartate receptor after neonatal brain ischemia. Ann Neurol. 63, 632-641.

24. Schwabe, R.F., Bradham, C.A., Uehara, T., Hatano, E., Bennett, B.L., Schoonhoven, R. and Brenner, D.A. (2003) c-Jun-N-terminal kinase drives cyclin D1 expression and proliferation during liver regeneration. Hepatology 37, 824-832

25. Pujol, M.J., Jaime, M., Serratosa, J., Jaumot, M., Agell, N. and Bachs, O. (2000) Differential association of p21Cip1 and p27Kip1 with cyclin E-CDK2 during rat liver regeneration. J. Hepatol. 33, 266-274

26. Li, W., Liang, X., Kellendonk, C., Poli, V. and Taub, R. (2002) STAT3 contributes to the mitogenic response of hepatocytes during liver regeneration. J. Biol. Chem. 277, 2841128417

27. Svegliati-Baroni, G., Ridolfi, F., Caradonna, Z., Alvaro, D., Marzioni, M., Saccomanno, S., Candelaresi, C., Trozzi ,L,. Macarri, G., Benedetti, A. and Folli, F. (2003) Regulation of ERK/JNK/p70S6K in two rat models of liver injury and fibrosis. J. Hepatol. 39, 528-537

28. Riehle, K.J., Campbell, J.S., McMahan, R.S., Johnson, M.M., Beyer, R.P., Bammler, T.K. and Fausto, N. (2008) Regulation of liver regeneration and hepatocarcinogenesis by suppressor of cytokine signaling 3. J. Exp. Med. 205, 91-103

29. Armeni, T., Ghiselli, R., Balercia, G., Goffi, L., Jassem, W., Saba, V. and Principato, G. (2000) Glutathione and ultrastructural changes in inflow occlusion of rat liver. J. Surg. Res. 88, 207-214

30. Rizzuto, R-, Bastianutto. C-, Brini, M., Murgia, M. and Pozzan, T. (1994) Mitochondrial Ca2+ homeostasis in intact cells. J Cell Biol. 126, 1183-1194

31. Sattler, M., Pride, Y.B., Ma, P., Gramlich, J.L., Chu, S.C., Quinnan, L.A., Shirazian, S., Liang, C., Podar, K., Christensen, J.G. and Salgia, R. (2003) A novel small molecule met inhibitor induces apoptosis in cells transformed by the oncogenic TPR-MET tyrosine kinase. Cancer Res. 63, 5462-5469

32. Tse, K.F., Allebach, J., Levis, M., Smith, B.D., Bohmer, F.D. and Small, D. (2002) Inhibition of the transforming activity of FLT3 internal tandem duplication mutants from AML patients by a tyrosine kinase inhibitor. Leukemia 16, 2027-2036

33. Burgel, P.R., Lazarus, S.C., Tam, D.C., Ueki, I.F., Atabai, K., Birch, M. and Nadel, J.A. (2001) Human eosinophils induce mucin production in airway epithelial cells via epidermal growth factor receptor activation. J. Immunol. 27, 5948-5954

34. Meydan, N., Grunberger, T., Dadi, H., Shahar, M., Arpaia, E., Lapidot, Z., Leeder, J.S., Freedman, M., Cohen, A., Gazit, A., Levitzki, A. and Roifman, C.M. (1996) Inhibition of acute lymphoblastic leukaemia by a Jak-2 inhibitor. Nature 379, 645-648.

35. Bain, J., McLauchlan, H., Elliott, M. and Cohen, P. (2003) The specificities of protein kinase inhibitors: an update. Biochem. J. 371, 199-204 
36. Oliver, J.M., Burg, D.L., Wilson, B.S., McLaughlin, J.L. and Geahlen, R.L. (1994) Inhibition of mast cell Fc epsilon R1-mediated signaling and effector function by the Syk-selective inhibitor, piceatannol. J. Biol. Chem. 269, 29697-29703

37. Akiyama, T. and Ogawara, T. (1991) Use and specificity of genistein as inhibitor of proteintyrosine kinases. Methods Enzymol. 201, 362-370

38. Engen JR, Wales TE, Hochrein JM, Meyn MA 3rd, Banu Ozkan S, Bahar I, Smithgall TE. (2008) Structure and dynamic regulation of Src-family kinases. Cell Mol Life Sci. 65, 30583073

39. Benati, D. and Baldari, C.T. (2008) SRC family kinases as potential therapeutic targets for malignancies and immunological disorders. Curr. Med. Chem. 15, 1154-1165.

40. Han, C., Bowen, W.C., Michalopoulos, G.K. and Wu, T. (2008) Alpha-1 adrenergic receptor transactivates signal transducer and activator of transcription-3 (Stat3) through activation of Src and epidermal growth factor receptor (EGFR) in hepatocytes. J. Cell. Physiol. 216, 486497

41. Chen, J. and Siddiqui, A. (2007) Hepatitis B virus X protein stimulates the mitochondrial translocation of Raf-1 via oxidative stress. J. Virol. 81, 6757-6760

42. Mayoral, R., Fernández-Martínez, A., Roy, R., Boscá, L. and Martín-Sanz, P. (2007) Dispensability and dynamics of caveolin-1 during liver regeneration and in isolated hepatic cells. Hepatology 46, 813-822

43. Griparic, L. and van der Bliek, A.M. (2005) Assay and properties of the mitochondrial dynamin related protein Opa1. Methods Enzymol. 404, 620-631

44. Sicheri, F., Moarefi, I., and Kuriyan, J. Crystal structure of the Src family tyrosine kinase Hck. Nature 385, 602-609.

45. Williams, J.C., Weijland, A., Gonfloni, S., et al. (1997) The 2.35 A crystal structure of the inactivated form of chicken Src: a dynamic molecule with multiple regulatory interactions. J. Mol. Biol. 274, 757-775.

46. Veracini, L., Franco, M., Boureux, A., Simon, V., Roche, S. and Benistant, C. (2006) Two distinct pools of Src family tyrosine kinases regulate PDGF-induced DNA synthesis and actin dorsal ruffles. J. Cell. Sci. 15, 2921-2934

47. Scapini P, Pereira S, Zhang H, Lowell CA.(2009) Multiple roles of Lyn kinase in myeloid cell signaling and function. Immunol Rev.228, 23-40

48. Sussman MA. (2009) Mitochondrial integrity: preservation through Akt/Pim-1 kinase signaling in the cardiomyocyte. Expert Rev Cardiovasc Ther. 7, 929-938.

49. Yonekawa H, Akita Y.(2009) Protein kinase Cepsilon: the mitochondria-mediated signaling pathway. FEBS J. 275, 4005-4913.

50. Boerner JL, Demory ML, Silva C, Parsons SJ. (2004) Phosphorylation of Y845 on the epidermal growth factor receptor mediates binding to the mitochondrial protein cytochrome $\mathrm{c}$ oxidase subunit II. Mol Cell Biol. 24,7059-7071.

51. Barksdale KA, Bijur GN. (2009) The basal flux of Akt in the mitochondria is mediated by heat shock protein 90. J Neurochem. 108, 1289-1299

52. Trentin L, Frasson M, Donella-Deana A, Frezzato F, Pagano MA, Tibaldi E, Gattazzo C, Zambello R, Semenzato G, Brunati AM. (2008) Geldanamycin-induced Lyn dissociation from aberrant Hsp90-stabilized cytosolic complex is an early event in apoptotic mechanisms in B-chronic lymphocytic leukemia. Blood.112, 4665-4674.

53. Feng, J., Zhu, M., Schaub, M.C., Gehrig, P., Roschitzki, B., Lucchinetti, E. and Zaugg, M. (2008) Phosphoproteome analysis of isoflurane-protected heart mitochondria: phosphorylation of adenine nucleotide translocator-1 on Tyr194 regulates mitochondrial function. Cardiovasc. Res. 80, 20-29 
B Biochemical Journal Immediate Publication. Published on 16 Oct 2009 as manuscript BJ20090902

\section{FUNDING}

Supported by grant from University of Padova to A.M. Brunati [Progetto di Ateneo 2005]. 


\section{FIGURE LEGENDS}

Figure 1. Protein tyrosine phosphorylation increases in isolated mitochondria from rat regenerating liver after PH. (A) Mitochondrial lysates from rat livers after $\mathrm{PH}$ at different timepoints were assayed by Western blot analysis with anti-phospho-tyrosine antibody. The membranes were reprobed with anti-aconitase antibody as loading control. The molecular weight markers are indicated on the left. (B) Total cell lysates from rat livers after PH at different time-points were assayed by Western blot analysis with anti-cyclin D1, anti-cyclin E1, anti-P-STAT3 and anti-PERK1/2 antibodies. The resulting bands underwent densitometric analysis (arbitrary units). The membranes were reprobed with anti- $\beta$-actin, anti-STAT3 and anti-ERK1/2 antibodies antibody as loading control. (C) Mitochondrial lysates from rat livers after $\mathrm{PH}$ at different time-points were tested for glutathione redox status (GSSG/GSH, top panel), $\mathrm{Ca}^{2+}$ content (middle panel) and membrane potential by monitoring the distribution of tetraphenylphosphonium across the mitochondrial membrane (bottom panel), as described in the section Materials and Methods. (D) Mitochondrial lysates (upper panel) and cytosol (lower panel) from rat livers after PH at different time-points were assayed by Western blot analysis with anti-cytochrome c antibody. The resulting bands underwent densitometric analysis (arbitrary units). The membranes were reprobed with antiaconitase and anti- $\beta$-actin antibodies as loading control. (E) Total cell lysates from rat livers after $\mathrm{PH}$ at different time-points were assayed by Western blot analysis with anti-PARP antibody. The membranes were reprobed anti- $\beta$-actin antibodies as loading control. All data shown are representative of 3 to 6 rats per time-point after $\mathrm{PH}$ and presented as mean $\pm \mathrm{SD}$.

Figure 2. Effect of TPK inhibitors on tyrosine kinase activities of total cell and mitochondrial lysates $24 \mathbf{~ h}$ after PH. $100 \mu \mathrm{g}$ of protein from total cell (A) and mitochondrial (B) lysates were tested for tyrosine kinase activity in the absence (Ctrl) and presence of SU11274 (2 $\mu \mathrm{M})$, AG1478 $(20 \mu \mathrm{M})$, AG1296 $(20 \mu \mathrm{M}), \mathrm{AG} 490(20 \mu \mathrm{M})$, PP2 $(7.5 \mu \mathrm{M})$, SU6656 (7.5 $\mu \mathrm{M})$, piceatannol (15 $\mu \mathrm{M})$ or genistein $(20 \mu \mathrm{M})$ respectively by using the nonspecific random polymer polyGlu $\mathrm{T}_{4} \mathrm{r}$ as a substrate. Data are representative of 3 experiments performed in triplicate and presented as mean \pm SD. $\mathrm{P}<0.05(*)$ and $\mathrm{P}<0.01(* *)$ are versus $\mathrm{Ctrl}$.

Figure 3. Lyn is the predominant SFK expressed in the liver. (A) $50 \mu \mathrm{g}$ of protein form total cell lysate of rat spleen, brain and liver were assayed by Western blot analysis with antibodies against each member of SFKs and the resulting bands were subjected to densitometric analysis (arbitrary units). Data are representative of 3 experiments performed in triplicate and presented as mean \pm SD. (B) The level of mRNAs coding for c-Src and Lyn from rat liver after $\mathrm{PH}$ at different time-points was analyzed by RT-PCR. The level of mRNA for $\beta$-actin was measured as an internal control.

Figure 4. Lyn localizes to mitochondria in an activated form. Comparable aliquots of (A) total cell and (B) mitochondrial lysates from rat liver after $\mathrm{PH}$ at different time-points were assayed for in vitro Lyn activity on Src-specific peptide substrate cdc2(6-20) and by Western blotting for Lyn. The membranes were reprobed with anti- $\beta$-actin and anti-aconitase antibodies, respectively, as loading control (C) Total cell and (D) mitochondrial lysates from rat liver after PH at different time-points were immunoprecipitated with anti-Lyn antibody, the immunoprecipitates were assayed with anti-pYA and, after stripping, with anti-Lyn antibody. The figure is representative of experiments performed in triplicate.

Figure 5. Subcellular localization of Lyn during LR after PH. $250 \mu$ g of liver homogenate at (A) 0, (B) 12 and (C) 24 hours after PH was subjected to differential centrifugation to separate the particulate fraction from nuclei and cytosol. The particulate fraction was further fractionated by centrifugation on discontinuous OptiPrep ${ }^{\mathrm{TM}}$ gradient to separate the cellular organelles as described 
in the section Material and Methods. Aliquots of the resulting fractions were assayed for Lyn activity tested on Src-specific peptide substrate cdc2(6-20), and analyzed by immunoblotting with anti-Lyn antibody. (D) Aliquots of the same fractions underwent immunoblotting with the following organelle-specific antibodies: anti-PMCA (plasma membrane), anti-GM130 (Golgi apparatus), anti-calnexin (ER) and anti-aconitase (mitochondria). The figure is representative of experiments performed in triplicate.

Figure 6. Lyn is detected as a soluble protein in the intermembrane space of mitochondria. (A) Mitochondria purified from rat liver 24h after $\mathrm{PH}$ were incubated in the absence or presence of proteinase $\mathrm{K}$ and Triton X-100 according to the protocol reported in the section Materials and Methods. Aliquots of mitochondrial lysate were subjected to Western blot analysis with anti-Lyn, anti-AIF and anti-aconitase antibodies, respectively. (B) Intact mitochondria, mitochondrial membranes and soluble fractions obtained from rat livers $24 \mathrm{~h}$ after $\mathrm{PH}$ and described in the section Materials and Methods were subjected to Western blot analysis with anti-Lyn, anti-AIF and antiaconitase antibodies, respectively. (C) Mitochondria purified from rat liver $24 \mathrm{~h}$ after $\mathrm{PH}$ were incubated in the presence of increasing concentrations of digitonin. After each treatment the samples were centrifuged to separate pellet $(\mathrm{P})$ from soluble fraction (S). Aliquots of such fractions after differential digitonin treatment were subjected to Western blot analysis with anti-Lyn, antiAIF and anti-aconitase antibodies, respectively. (D) The soluble fraction of mitochondria purified from rat liver $24 \mathrm{~h}$ after $\mathrm{PH}$ was loaded on top of a linear glycerol gradient (10-40\%) and centrifuged $18 \mathrm{~h}$ at $100000 \mathrm{~g}$ in a SW60Ti rotor (Beckman) at $4^{\circ} \mathrm{C} .18$ fractions $(200 \mu \mathrm{L}$ each) were collected from top and analyzed by Western blot analysis with anti-Lyn antibody. The figure is representative of experiments performed in triplicate. Downward arrows: position of molecular weight standards on glycerol gradients, glutamate dehydrogenase $(62 \mathrm{kDa})$, alcohol dehydrogenase $(150 \mathrm{kDa})$, apoferritin $(443 \mathrm{kDa})$ and thyroglobulin $(669 \mathrm{kDa})$ (Sigma-Aldrich) are indicated to estimate the molecular weight of the protein complexes on parallel gradient runs.

Figure 7. Effect of tyrosine phosphatase inhibitors on mitochondrial membrane potential $(\Delta \Psi \mathbf{m})$. Mitochondria were purified from equivalent amounts of both quiescent and regenerating rat liver $24 \mathrm{~h}$ after $\mathrm{PH}$ in the presence or absence of tyrosine phosphatase inhibitors (PTP-I). (A) Mitochondrial aliquots from both quiescent (lanes 1 and 2) and regenerating (lanes 3 and 4) rat liver $24 \mathrm{~h}$ after $\mathrm{PH}$ in the presence (lanes 1 and 3) or absence (lanes 2 and 4) of tyrosine phosphatase inhibitors (PTP-I) were subjected to Western blot analysis with anti-phosphotyrosine antibody and the membranes were reprobed with anti-aconitase antibody as loading control. The molecular weight markers are indicated on the right. (B) $\Delta \Psi \mathrm{m}$ was measured as described in [14] on mitochondrial aliquots from both quiescent (left panel) and regenerating (right panel) rat liver $24 \mathrm{~h}$ after PH in the presence or absence of tyrosine phosphatase inhibitors (PTP-I), incubated in standard medium. The downward arrows indicate the time-point at which ADP was added (final concentration $200 \mu \mathrm{M}$ ). The figure is representative of experiments performed in triplicate on 3 samples from both quiescent and regenerating livers. $\Delta \mathrm{E}$, electrode potential.

Figure 8. Effects of PP2 treatment of rats on LR 24h after PH. (A) Mitochondrial lysates obtained $24 \mathrm{~h}$ after $\mathrm{PH}$ from liver of rats treated with either increasing concentrations of PP2 or vehicle were assayed by Western blot analysis with anti-phospho-tyrosine antibody. The membranes were reprobed with anti-aconitase antibody as loading control. The molecular weight markers are indicated on the left. (B) Total cell (upper panel) and mitochondrial (lower panel) lysates obtained 24h after PH from liver of rats treated with either increasing concentrations of PP2 or vehicle rat liver were immunoprecipitated with anti-Lyn antibody, the immunoprecipitates were assayed with anti-pYA and, after stripping, with anti-Lyn antibody. (C) $\Delta \Psi \mathrm{m}$ was measured as described in [14] on mitochondria obtained $24 \mathrm{~h}$ after $\mathrm{PH}$ from liver of rats treated with either 
increasing concentrations of PP2 or vehicle incubated in standard medium. The downward arrows indicate the time-point at which ADP was added (final concentration $200 \mu \mathrm{M}$ ). $\Delta \mathrm{E}$, electrode potential. (D) Cytosol obtained $24 \mathrm{~h}$ after $\mathrm{PH}$ from liver of rats treated with either increasing concentrations of PP2 or vehicle were assayed by Western blot analysis with anti-cytochrome c, anti-aconitase and anti-LDH respectively. The figure is representative of experiments performed in triplicate. 
Fig. 1

A

Time after PH (h) $\quad 0 \quad 12 \quad 24 \quad 36 \quad 48$

B

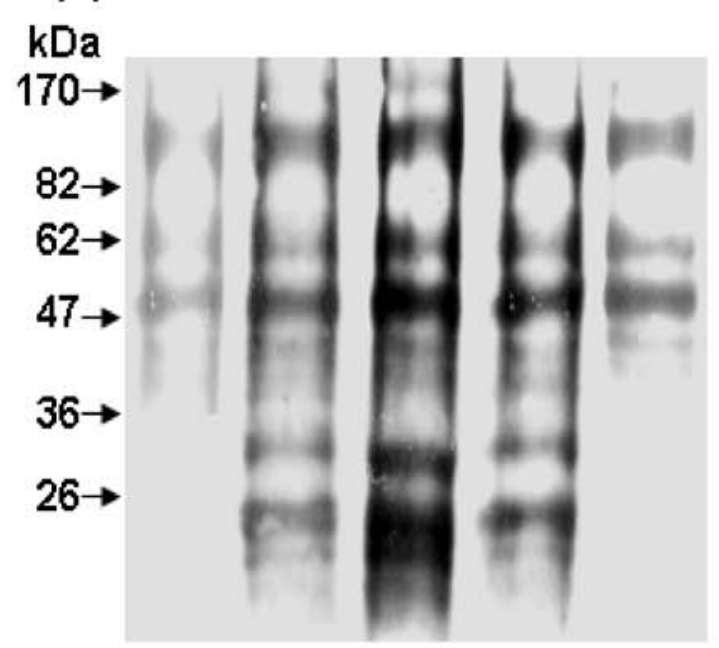

Wb: aconitase

Wb: pTyr

$$
\begin{gathered}
\mathrm{kDa} \\
170 \rightarrow \\
82 \rightarrow \\
62 \rightarrow
\end{gathered}
$$

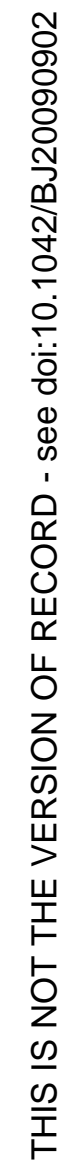

$\begin{array}{llllll}\text { Time after } \mathrm{PH}(\mathrm{h}) & 0 & 12 & 24 & 36 & 48\end{array}$
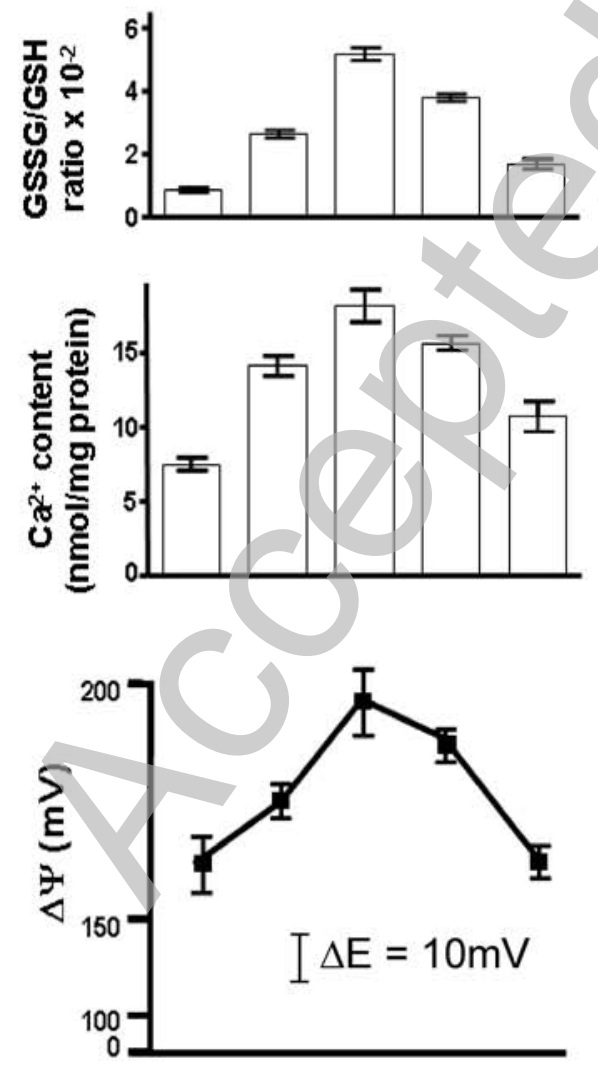

$\begin{array}{llllll}\text { Time after PH (h) } & 0 & 12 & 24 & 36 & 48\end{array}$

Wb: cyclin D1
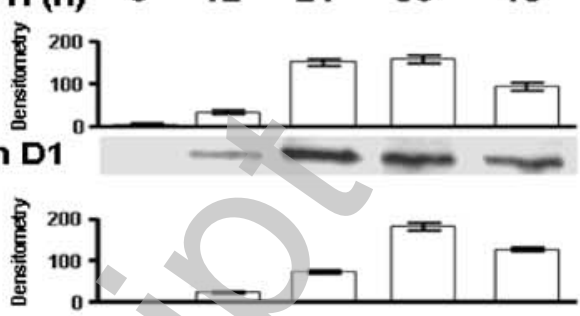

Wb: cyclin E1

Wb: $\beta$-actin

Wb: P-STAT 3

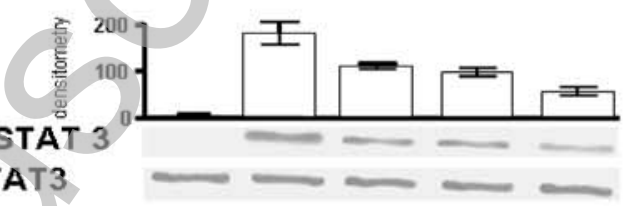

Wb: STAT3

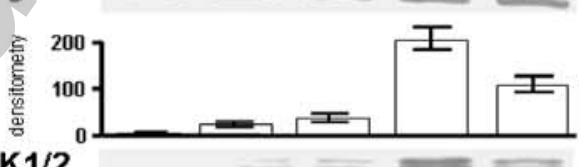

Wb: P-ERK1/2

Wb: ERK1/2

D

$\begin{array}{llllll}\text { Time after PH (h) } & 0 & 12 & 24 & 36 & 48\end{array}$

Mitochondria

Wb: cytochrome c $-\infty-$ $\mathrm{Wb}$ : aconitase

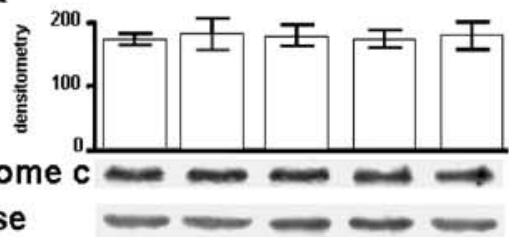

Cytosol

Wb: cytochrome $\mathrm{c}$

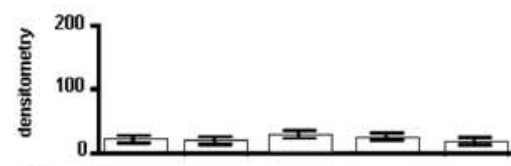

Wb: $\beta$-actin

$\mathbf{E}$

$\begin{array}{llllll}\text { Time after } \mathrm{PH}(\mathrm{h}) & 0 & 12 & 24 & 36 & 48\end{array}$ $\mathrm{kDa}$

Wb: PARP

$116 \rightarrow-\infty-$

$89 \rightarrow$

Wb: $\beta$-actin

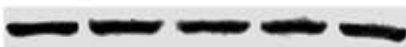


Fig. 2
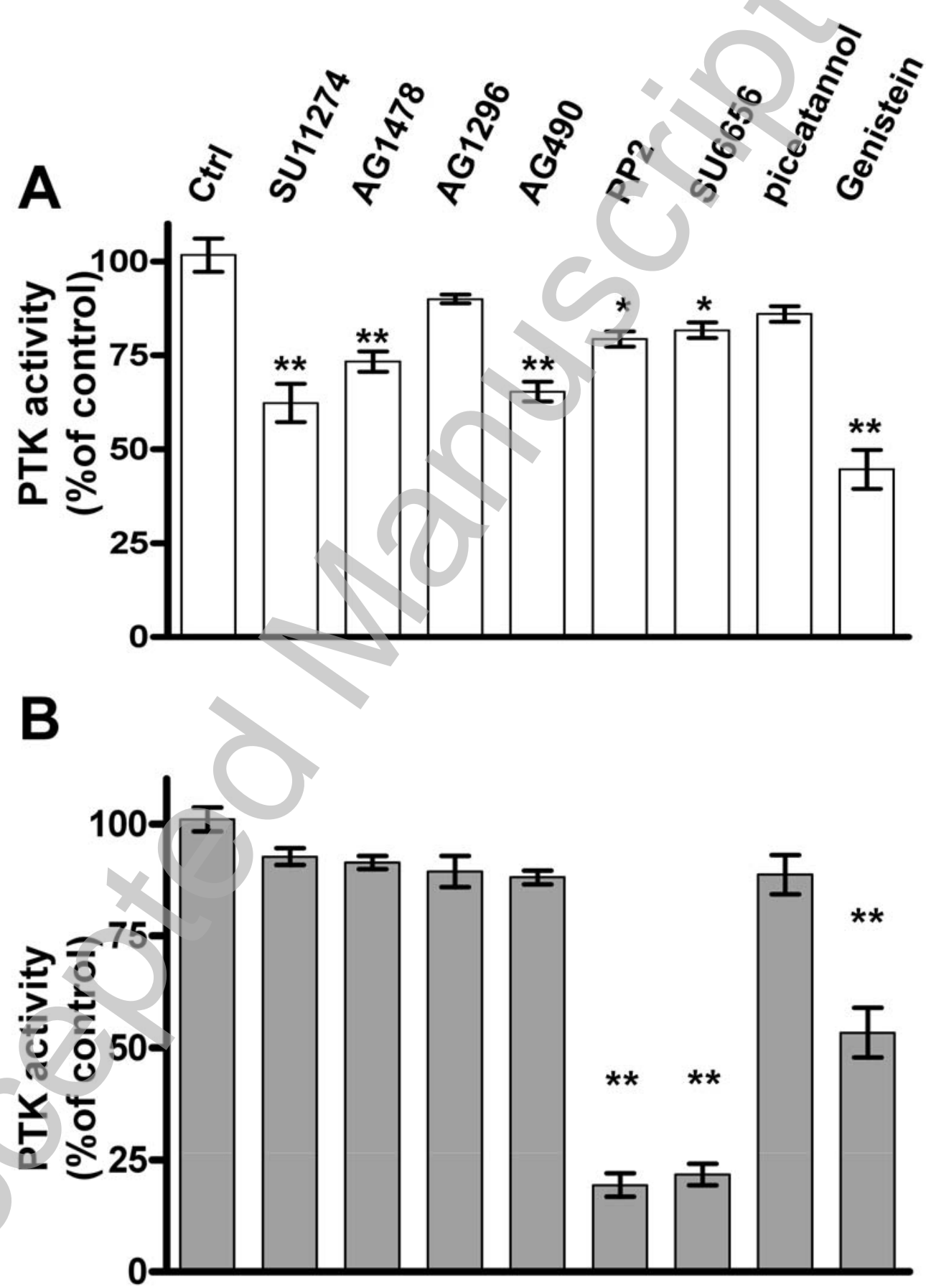
Biochemical Journal Immediate Publication. Published on 16 Oct 2009 as manuscript BJ20090902

Fig. 3

A

Src releted
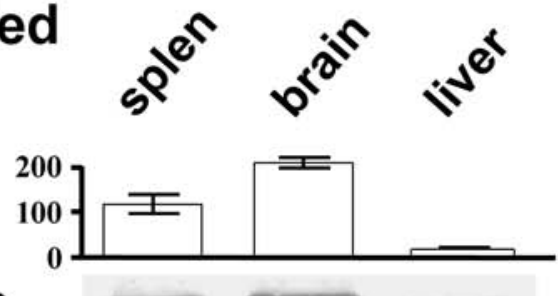

Wb: c-Src

Wb: Yes

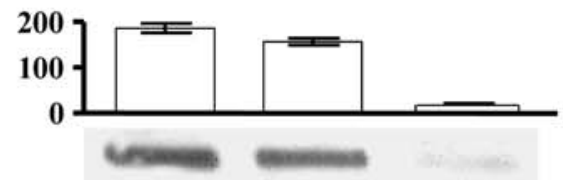

Wb: Fyn

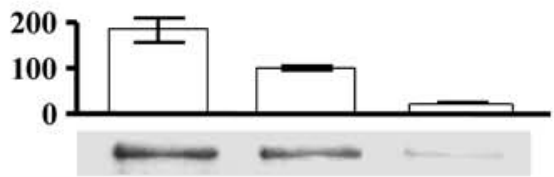

Wb: Fgr

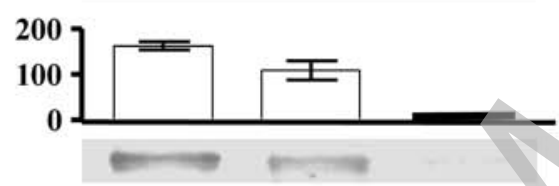

Wb: $\beta$-actin
Lyn releted

Wb: Lyn

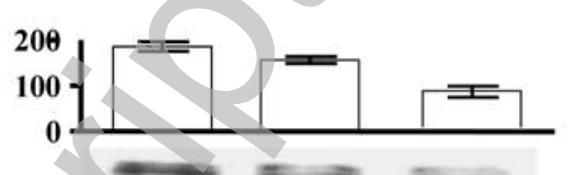

Wb: Lck

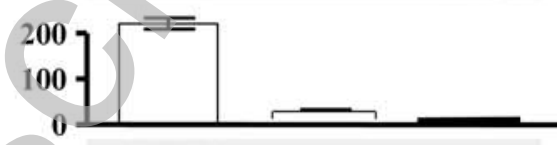

Wb: Hck

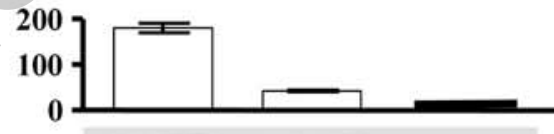

Wb: Blk

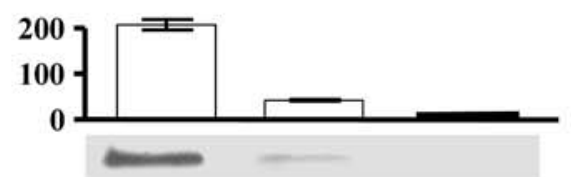

Wb: $\beta$-actin

\section{$\begin{array}{llllll}\text { Time after PH (h) } & 0 & 12 & 24 & 36 & 48\end{array}$}

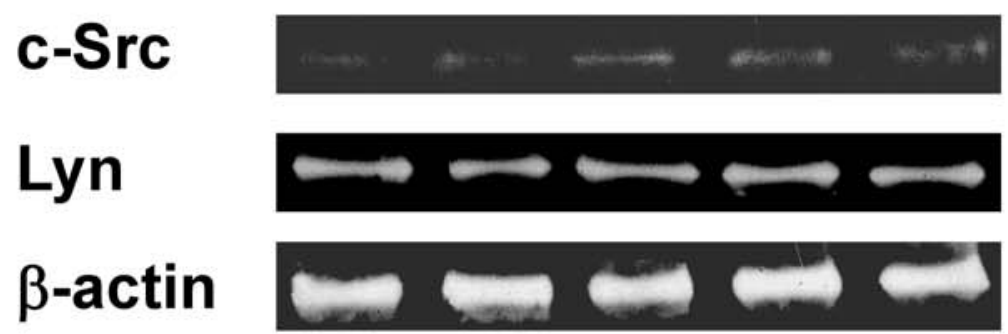


Fig. 4

A

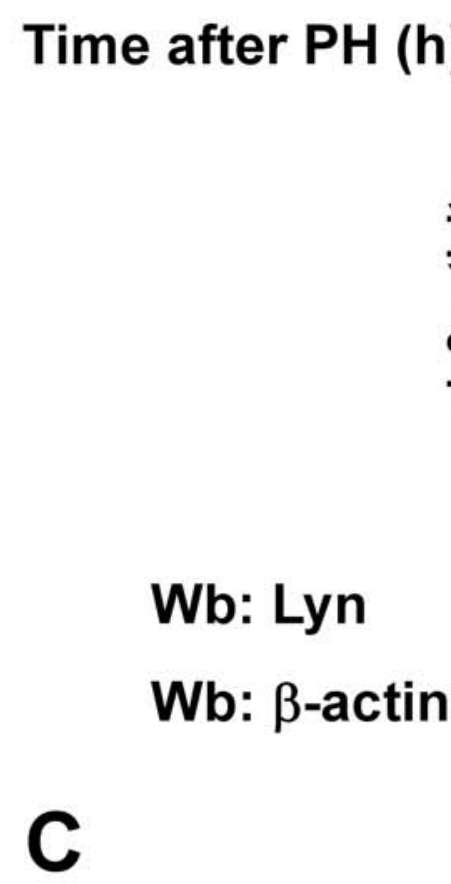

\section{Ip: Lyn}

\section{Time after $\mathrm{PH}(\mathrm{h})$}

Wb: $P-Y_{A}$

Wb: Lyn
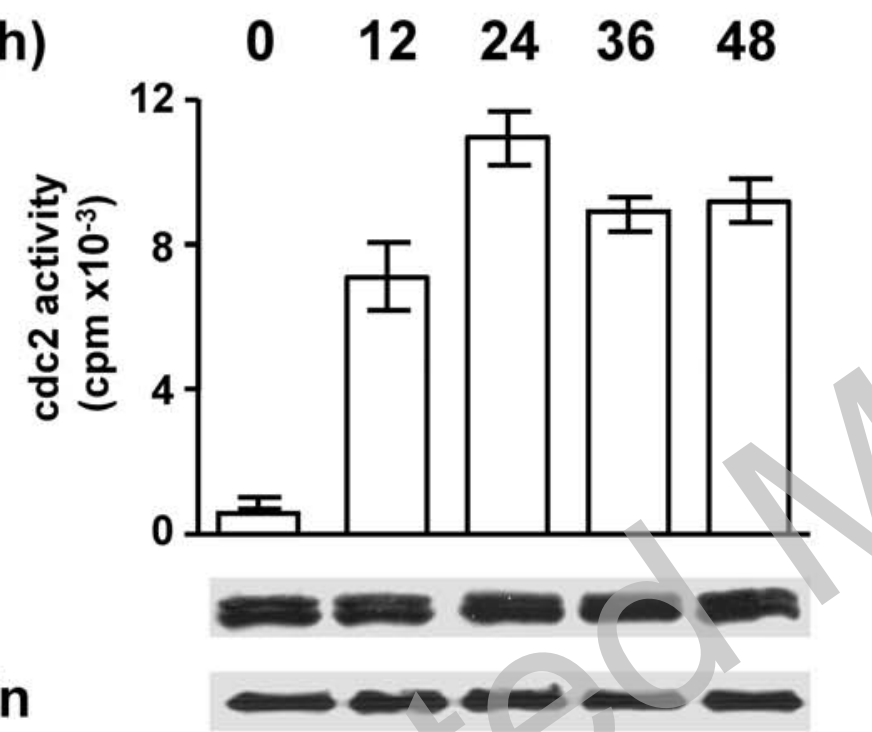

Wb: Lyn

$\mathrm{Wb}$ : aconitase

D

\section{Ip: Lyn}

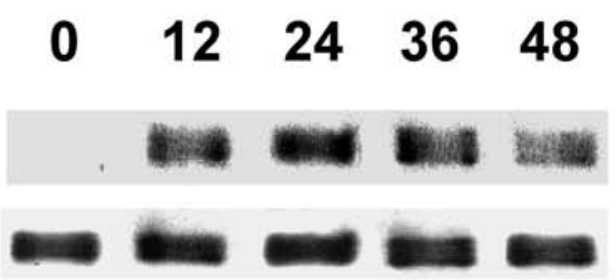

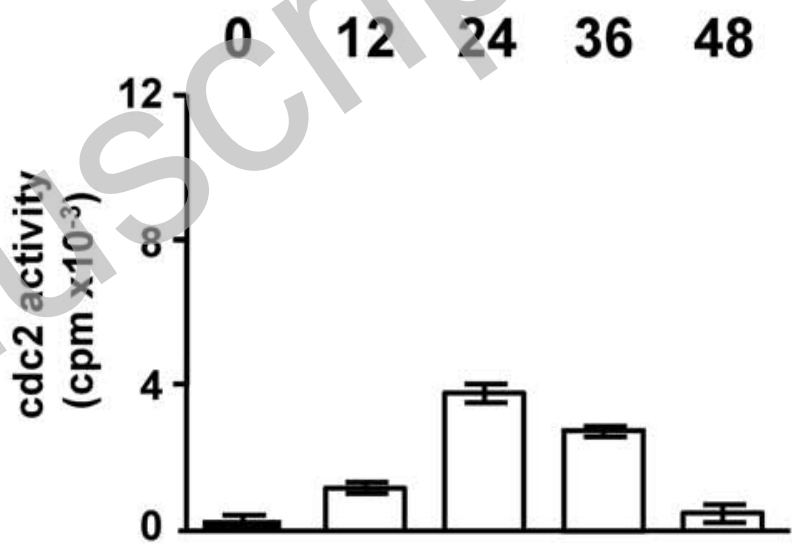

-

$\begin{array}{lllll}0 & 12 & 24 & 36 & 48\end{array}$

Wb: $P-Y_{A}$

Wb: Lyn 
Fig. 5

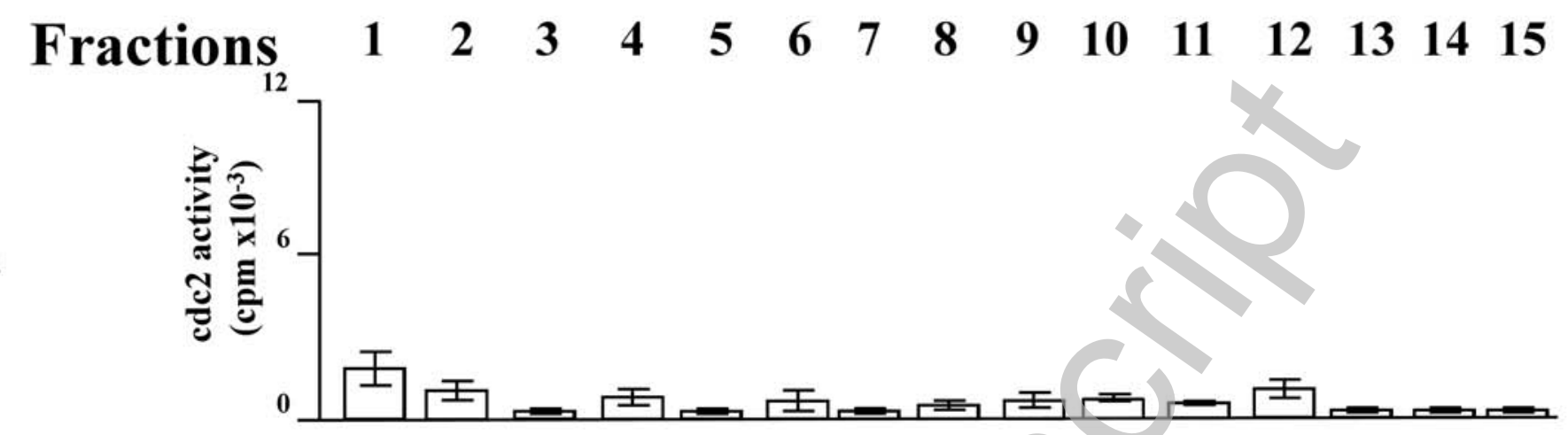

Wb:Lyn

B

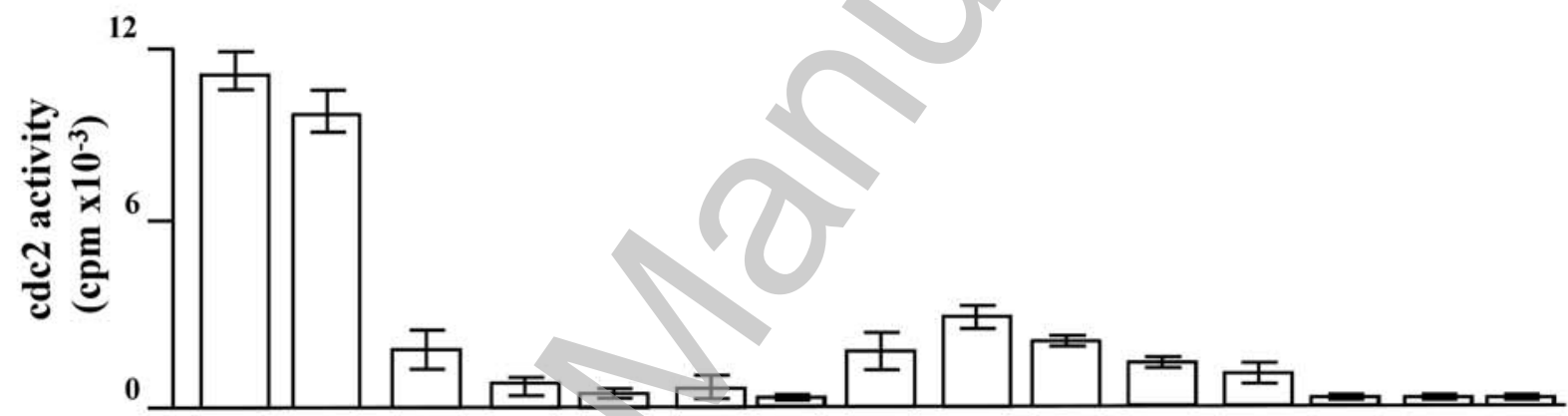

Wb:Lyn

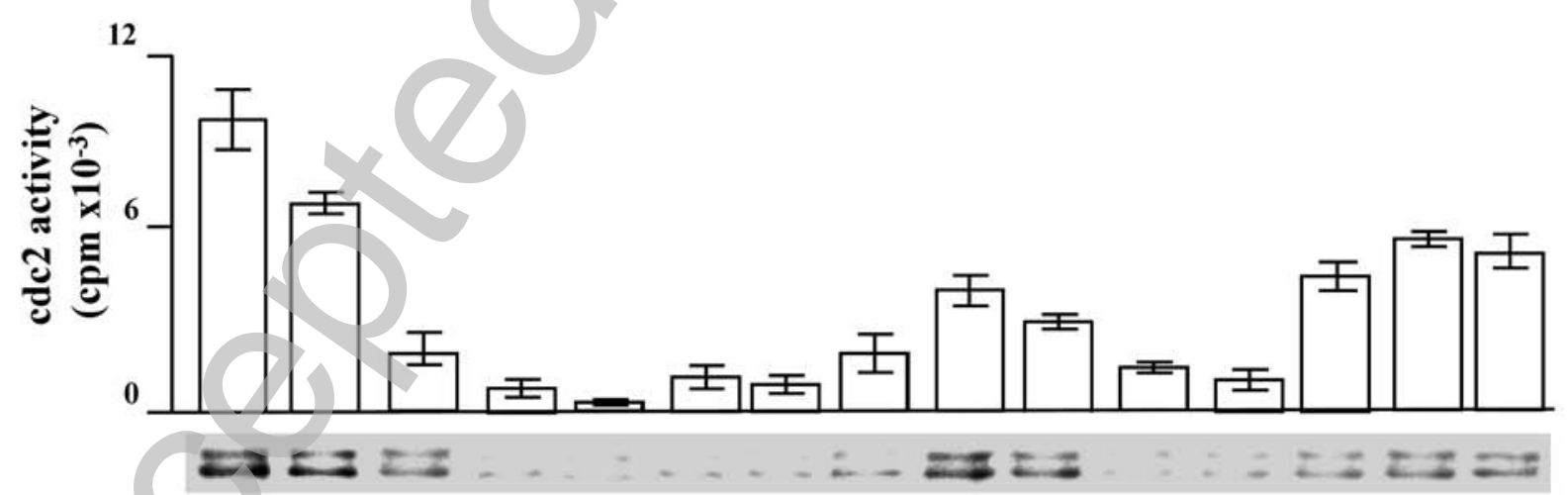

Wb: PMCA

Wb: GM130

Wb: Calnexin

Wb: aconitase 
B Biochemical Journal Immediate Publication. Published on 16 Oct 2009 as manuscript BJ20090902

\section{Fig. 6}

A

Wb: Lyn

Wb: AIF

PK

Tx-100
Wb: aconitase
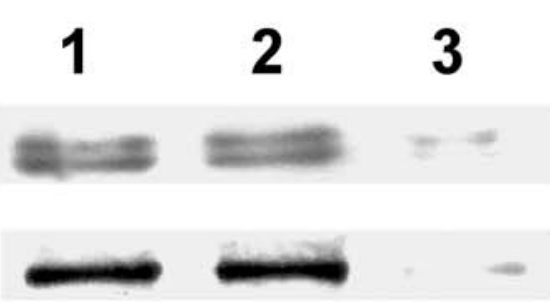

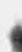

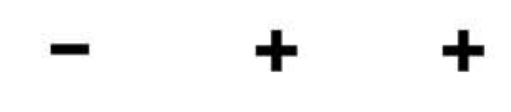

$-\quad-$

B

$$
123
$$

Wb: Lyn

Wb: AIF

Wb: aconitase

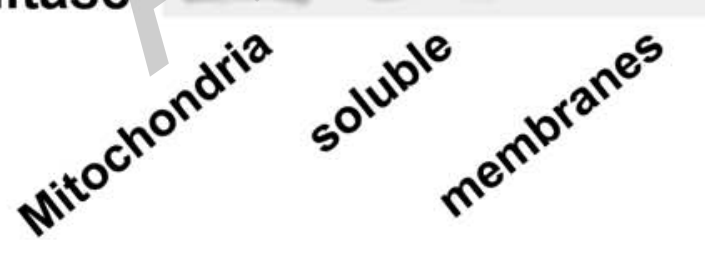

C

Wb: Lyn

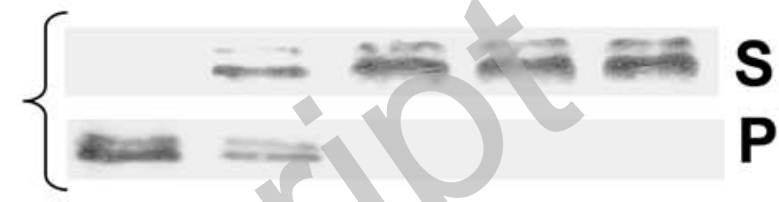

Wb: AIF

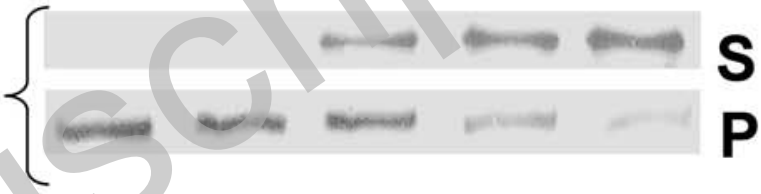

Wb: aconitase

$\begin{array}{llllll}\text { Digitonin (mg/ml) } & 0 & .10 & .20 & .40 & .60\end{array}$ $\mathbf{P}$

\section{P \\ S $P$ \\ S}


A

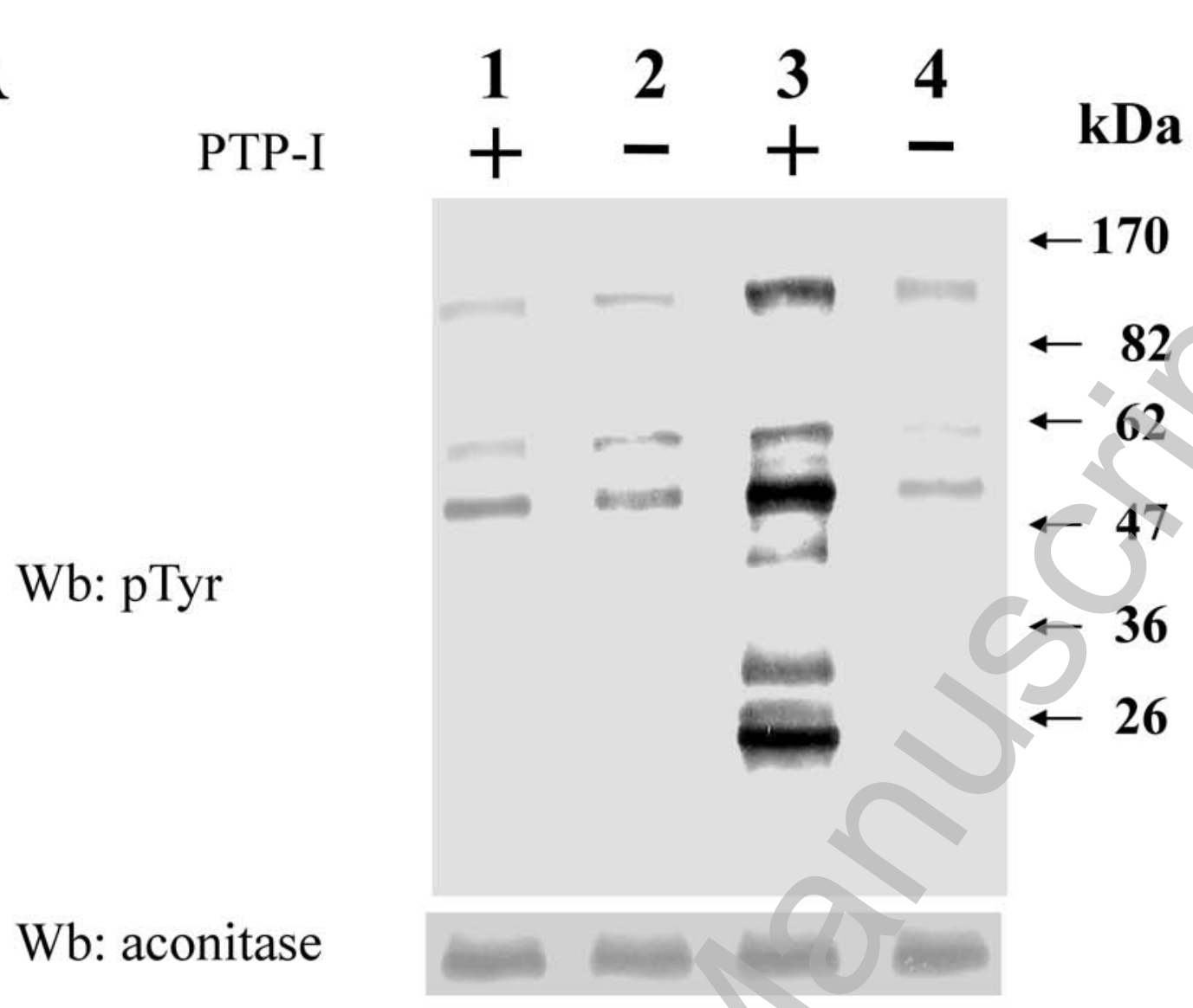

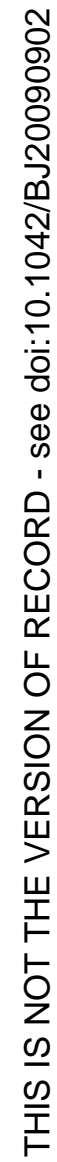

$\mathrm{Wb}$ : aconitase

B

$\downarrow$ ADP
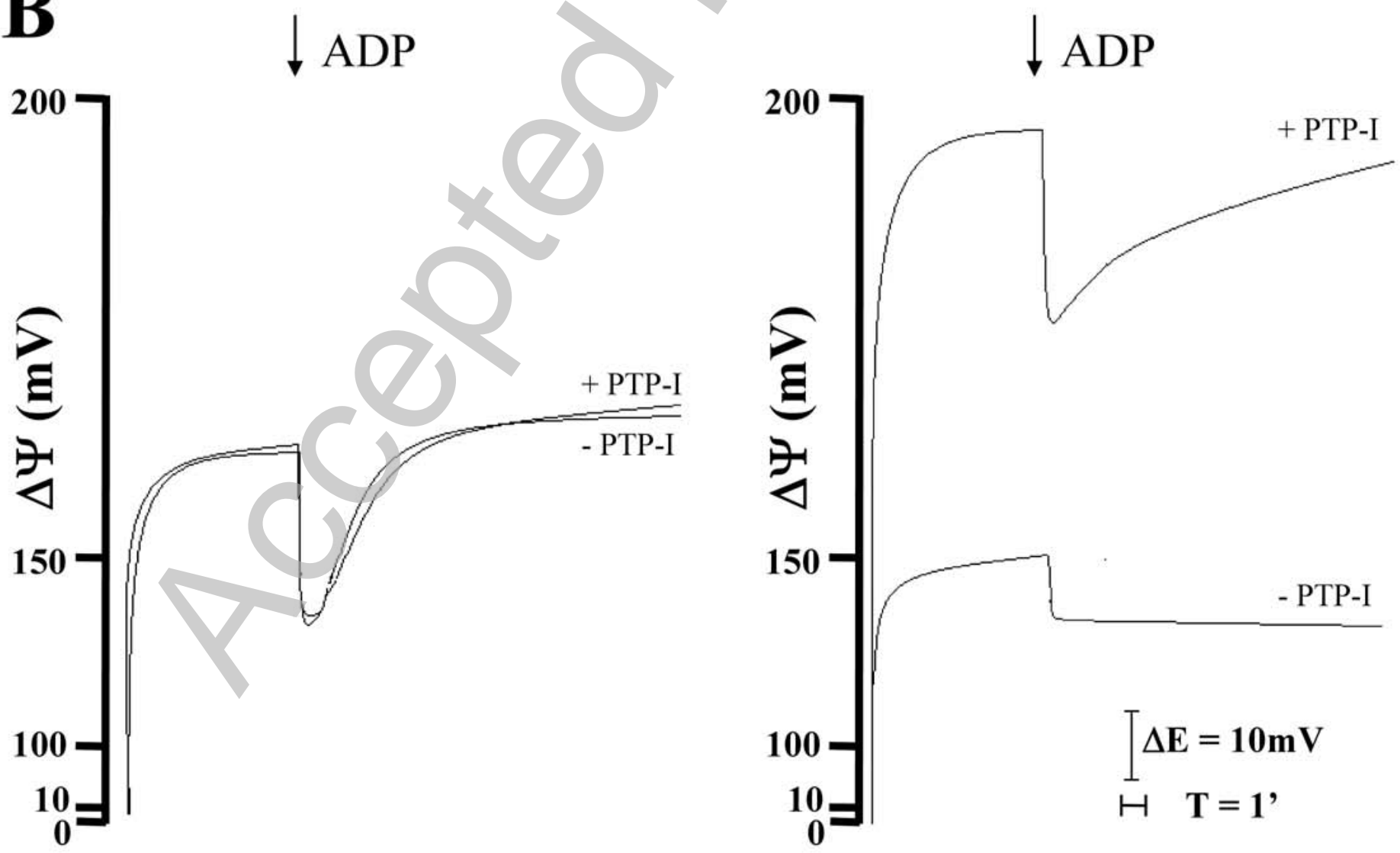

Licenced copy. Copying is not permitted, except with prior permission and as allowed by law. (C) 2009 The Authors Journal compilation (c) 2009 Portland Press Limited 
Fig.8

A

$\begin{array}{llll}1 & 2 & 3 & 4\end{array}$

B

$\begin{array}{lllll}\text { PP2 }(\mu \mathrm{g} / \mathrm{gm}) & - & 0.2 & 0.5 & 1\end{array}$

PP2 ( $\mu$ g/gm) $\quad-\quad 0.2 \quad 0.51$

kDa

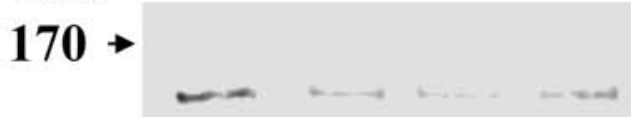

Wb:pTyr $47 \rightarrow \square+0$

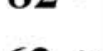

$62+$
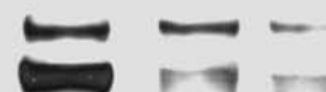

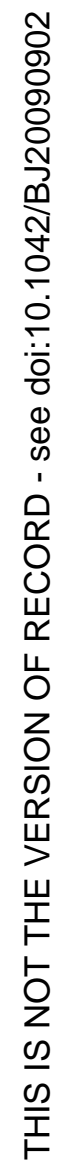

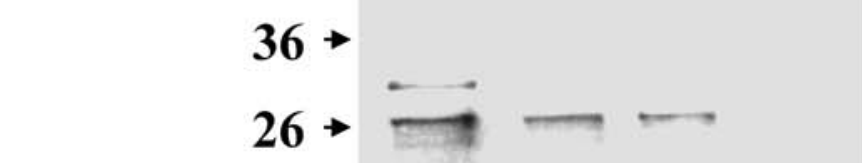

Wb: aconitase

Lysate

Ip: Lyn

Wb: P-Y

Wb: Lyn

$\begin{array}{llll}1 & 2 & 3 & 4\end{array}$

Mitochondria

Ip: Lyn

$\mathbf{W b}: \mathbf{P}-\mathbf{Y}_{\mathrm{A}}$

Wb: Lyn
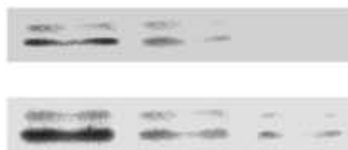

D

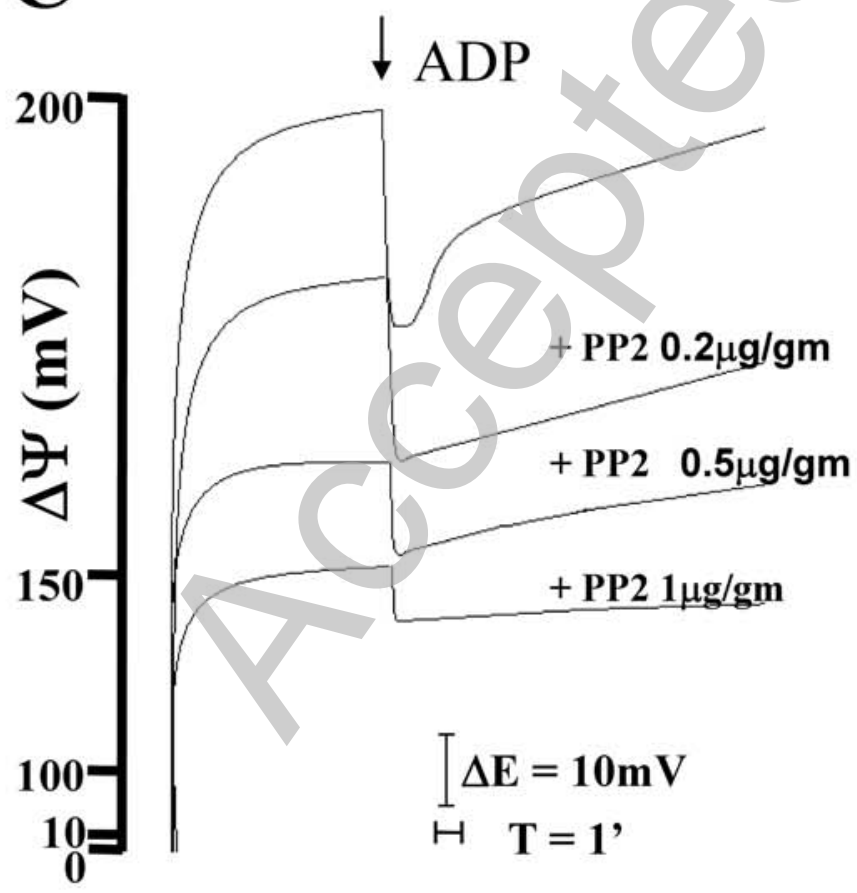

$\begin{array}{llll}1 & 2 & 3 & 4\end{array}$

$\begin{array}{lllll}\text { PP2 }(\mu \mathrm{g} / \mathrm{gm}) & - & 0.2 & 0.5 & 1\end{array}$

\section{Cytosol}

Wb: cytochrome c

Wb: aconitase

Wb: LDH

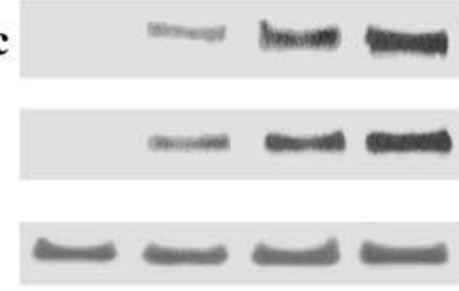

Licenced copy. Copying is not permitted, except with prior permission and as allowed by law.

(C) 2009 The Authors Journal compilation (c) 2009 Portland Press Limited 